Portland State University

PDXScholar

$11-27-1991$

\title{
Development of an Advisory System for Indoor Radon Mitigation
}

Patricia Hiromi Byrne

Portland State University

Follow this and additional works at: https://pdxscholar.library.pdx.edu/open_access_etds

Part of the Mechanical Engineering Commons

Let us know how access to this document benefits you.

\section{Recommended Citation}

Byrne, Patricia Hiromi, "Development of an Advisory System for Indoor Radon Mitigation" (1991).

Dissertations and Theses. Paper 4263.

https://doi.org/10.15760/etd.6147

This Thesis is brought to you for free and open access. It has been accepted for inclusion in Dissertations and Theses by an authorized administrator of PDXScholar. Please contact us if we can make this document more accessible: pdxscholar@pdx.edu. 
AN ABSTRACT OF THE THESIS OF Patricia Hiromi Byrne for the Master of Science in Mechanical Engineering presented November 27,1991 .

Title: Development of an Advisory System for Indoor Radon Mitigation.

APPROVED BY THE MEMBERS OF THE THESIS COMMITTEE:
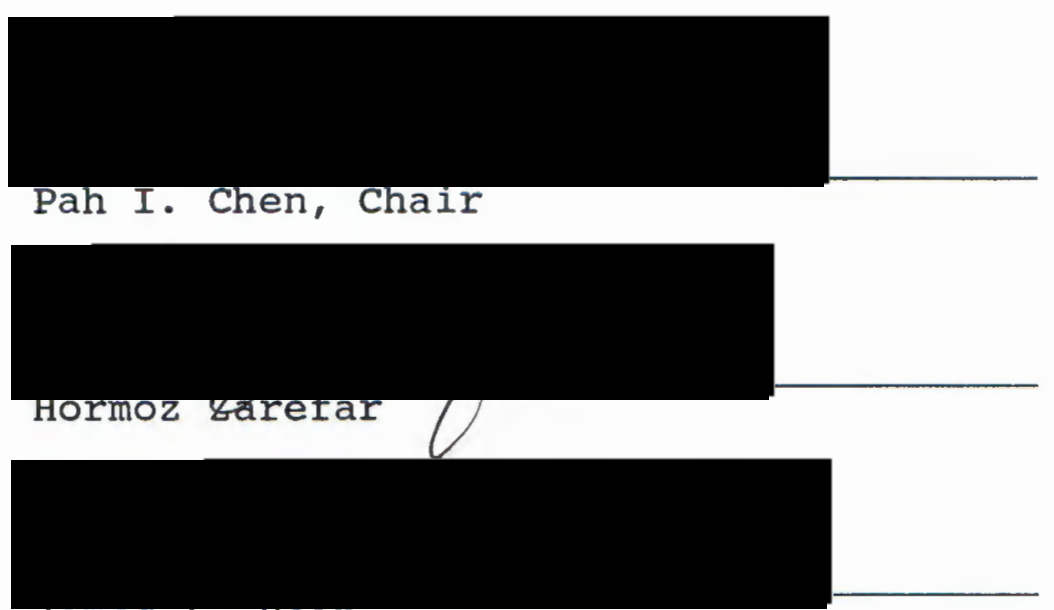

James L. Heln

A prototype hybrid knowledge-based advisory system for indoor radon mitigation has been developed to assist Pacific Northwest mitigators in the selection and design of mitigation systems for existing homes. The advisory system employs a heuristic inferencing strategy to determine which mitigation techniques are applicable, and applies procedural methods to perform the fan selection and cost estimation for particular techniques. The rule base has been developed employing knowledge in existing publications on radon 
mitigation. Additional knowledge has been provided by field experts. The benefits of such an advisory system include uniform record-keeping and consistent computations for the user, and verification of approved radon mitigation methods. 
DEVELOPMENT OF AN ADVISORY SYSTEM FOR INDOOR RADON MITIGATION

$$
\text { by }
$$

PATRICIA HIROMI BYRNE

A thesis submitted in partial fulfillment of the requirements for the degree of

\author{
MASTER OF SCIENCE \\ in \\ MECHANICAL ENGINEERING
}

Portland State University

1992 
TO THE OFFICE OF GRADUATE STUDIES:

The members of the committee approve the thesis of Patricia Hiromi Byrne presented November 27, 1991.
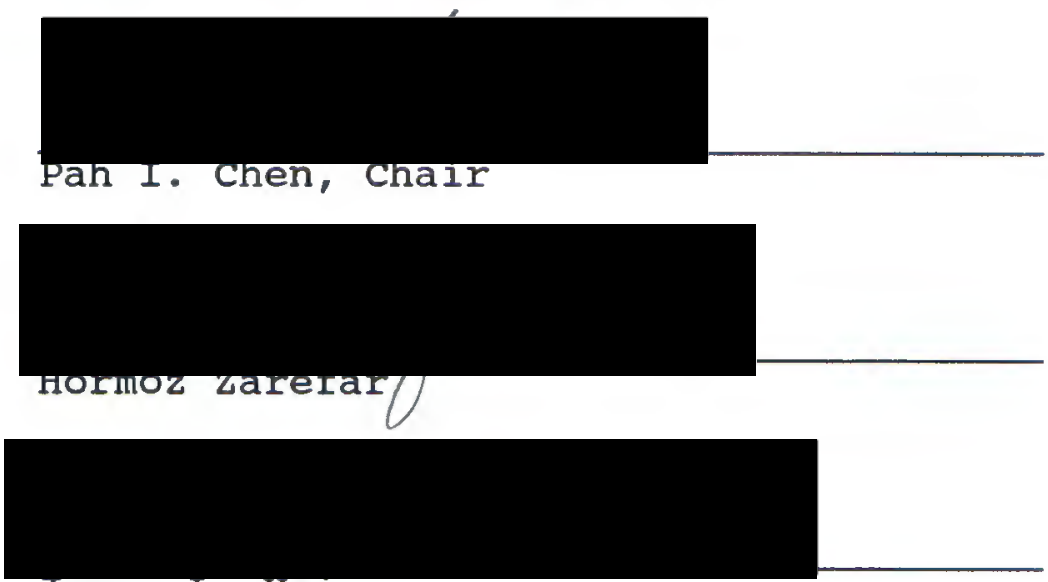

James L. Hein

APPROVED :

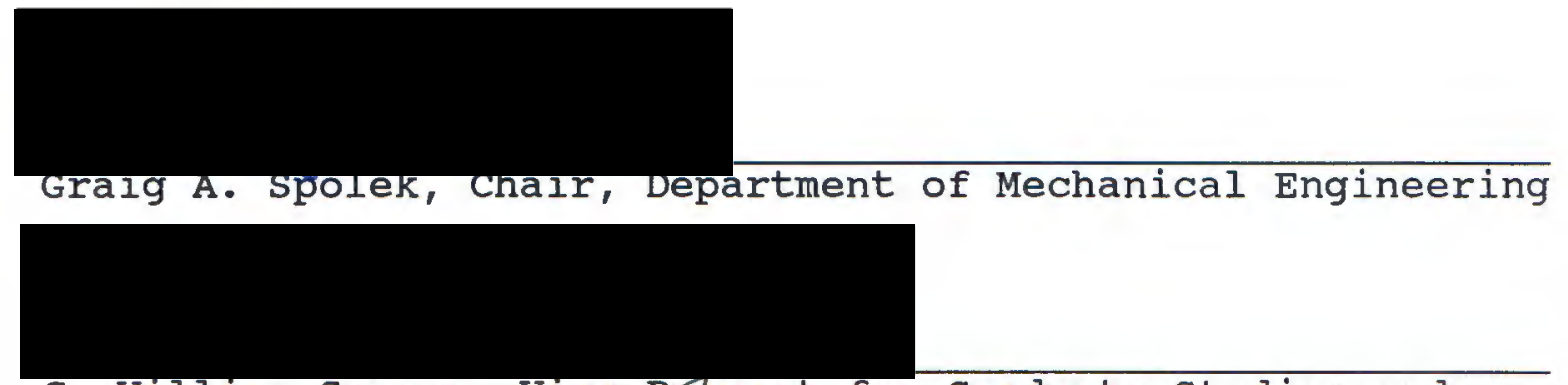

C. William Savery, Vice Pfovost for Graduate Studies and Research 


\section{ACKNOWLEDGEMENTS}

I wish to express my gratitude to all of the individuals who assisted in this project. My professors, Dr. Pah I. Chen and Dr. Hormoz Zarefar, were always capable of furnishing advice. A special thanks to Chuck Eastwood of Bonneville Power Administration for his constant dedication to the project, and all of the mitigation contractors, scientists, and EPA officials who have provided or will be providing expert opinion.

This work has been supported by Bonneville Power Administration, under research contract IAG-07967. 
TABLE OF CONTENTS

PAGE

ACKNOWLEDGEMENTS ..............................

LIST OF FIGURES ..........................

INTRODUCTION $\ldots \ldots \ldots \ldots \ldots \ldots \ldots \ldots \ldots \ldots \ldots \ldots \ldots \ldots \ldots$

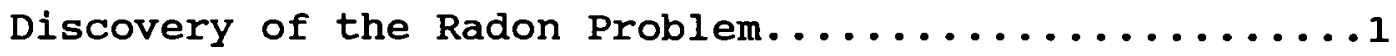

Initial Efforts to Combat Indoor Radon............

Radon Measurement and Reduction Methods...........4

A Solution to the Radon Problem................

KNOWLEDGE-BASED SYSTEMS ...................

Expert Systems Applications................

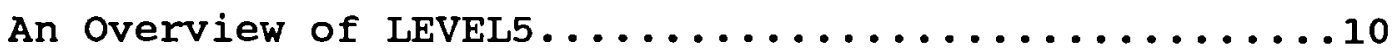

Inferencing strategies................... 15

Radon Mitigation and Knowledge-based Systems.......16

SYSTEM DEVELOPMENT. ......................... 17

General system structure................... 17

House Investigation Summary ..................... 19

Data Conversion and Mitigation Method Selection.....23

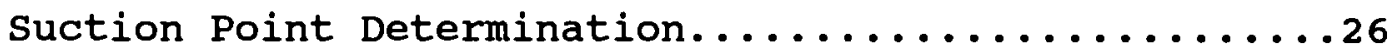

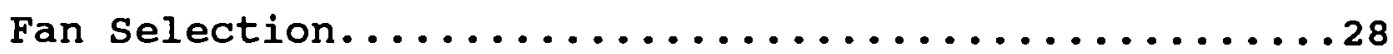

Cost Estimation.......................40

Fan Tutorial.........................

SUMMARY................................ 45 
Benefits to Radon Mitigators................ 45 Future Potential...................... 47 Projected work........................48 REFERENCES . 49 APPENDICES

A RNX2 . KNB SELECTED DEMONS AND METHODS......... 52

B FAN. KNB SELECTED DEMONS................60 


\section{LIST OF FIGURES}

1. Major components of an expert system.........9

2. A sample branch of the knowledge tree.........11

3. Classes, attributes, and instances...........13

4. An example of the graphical tools employed......14

5. A representation of the system modules........18

6. A portion of the working knowledge..........21

7. The recommended mitigation methods..........25

8. Suction point recommendation.............27

9. Data requested for ducting branches..........30

10. Diagnostic measurements requested.............31

11. Generic fan requirements...............32

12. Information on duct velocity.............38

13. Brand fans capable of meeting requirement.....40

14. Default costs for ducting..............41

15. Other costs used in estimation............42

16. Information presented in tutorial..........44 


\section{INTRODUCTION}

\section{DISCOVERY OF THE RADON PROBLEM}

In December of 1984, a Pennsylvania resident, stanley Watras, triggered the radiation alarms at the nuclear power plant where he worked. Subsequently it was discovered that the radon concentration in his home was so elevated that the health risk was equivalent to receiving 200,000 chest $\mathrm{x}$-rays per year (1). Radon exposure has been known to be a health risk, but was previously associated with uranium mining. only recently has it been understand that homes could have high radon concentrations resulting from the accumulation of radon produced by the decay of uranium in the soil.

Residents of the Reading Prong area of Pennsylvania were informed that radon produced in this manner was seeping up through their floors and accumulating in their homes (2). Further studies reveal that the Reading Prong area was not the only location with a radon problem. There is a global threat to indoor air quality from radon contamination, existing not only in the U.S., but in other countries as well (3). The severity of the radon problem varies widely. In the Pacific Northwest, $95 \%$ of the homes that participated in the Bonneville Power Administration (BPA) conservation program have concentration levels below 4 picocuries per 
Liter. This is the current "action level" recommended by the U.S. Environmental Protection Agency (EPA). At concentrations above this level, the EPA recommends that action be taken to reduce the concentration. Depending upon the degree of the concentration, action need not be immediate. The majority of homes in the Pacific Northwest have low concentrations, but the spokane/Coeur d'Alene area has a higher than average level, and there are a few homes in the Portland area with elevated levels of radon (4). Radon gas alone poses very little health threat. Since it is an inert gas, it will not react chemically. It can be breathed in and out of the lungs, and the chances of radioactive exposure are small due to the length of the half-life ( 3.8 days). The danger is due to the radon decay products (polonium 218, lead 214, bismuth 214, and polonium 214), which are created and decayed in less than an hour. Also, these elements are not inert. The atoms are capable of reacting with lung tissue or dust or other particles which can become lodged in lung tissue where they will continue to decay. People have just recently become aware that the decay of these by-products of radon can cause lung cancer in this manner, so there is very little data to assess the risk due to elevated levels in the home. However, there are estimates of the risks based on studies performed upon uranium miners who were exposed to varying levels of radon during their work. The studies revealed 
that prolonged exposure to radon and its decay products increases the risk of developing lung cancer (5). The longer the exposure and the higher the concentration, the greater the risk. Short term exposures to high levels are considered safer than long term exposures to lower levels. It is expected that radon will cause from 5,000 to 30,000 lung cancer deaths each year (2). Concern about the health risks has prompted government agencies to learn more about the actual health risks, how to measure radon levels, how to remove radon once it enters a home, and how to keep it from getting into the house (6).

\section{INITIAL EFFORTS TO COMBAT INDOOR RADON}

In 1986 the Environmental Protection Agency began publishing information to educate the public about the risks of radon exposure, and what they could do to diminish it. The 1988 EPA publication "Application of Radon Reduction Methods" by Mosley and Henschel (7) and "Practical Radon Control for Homes" by Brennan and Galbraith (8), explain and illustrate many radon mitigation strategies. These strategies can be grouped into two main classifications; those that attempt to prevent radon from entering the home, and those that dilute the radon once it has entered the home. Studies have been conducted at Lawrence Berkeley Laboratory (9) to determine the relation between the concentration levels and driving forces, and BPA (4) has 
conducted research to determine how the weatherization of homes and geological factors may affect the levels of indoor radon.

\section{RADON MEASUREMENT AND REDUCTION METHODS}

The existence of indoor radon is determined by taking screening and follow-up measurements. Measurement protocol calls for "closed house" conditions during a test in order to get the worst possible conditions. Since radon itself is an inert gas, it cannot be detected by normal chemical methods. The alpha particles released during decay are measured instead. A screening measurement is short-term, and is usually performed using a charcoal canister. This device is left in the home for several days to a week and then analyzed at a laboratory. If the result shows the radon level to be greater than the EPA action level, it is best to perform follow-up measurements. Follow-up measurements are long term, and are typically performed using a device called an alpha track detector. This device records the traces of alpha particles on a small strip of film, which is analyzed in a lab. Other measurement methods include grab samples and continuous radon monitoring. Grab samples, which are taken over a period of several minutes are useful as diagnostics, but are not appropriate for screening or follow-up measurements. Continuous radon monitoring is sometimes used for screening. 
The selection of a radon reduction system depends upon a wide collection of information such as initial radon level, geological factors, climate, house and site characteristics, driving forces, and possible entry routes. After a system is selected, the design and installation depends upon the mitigation method that was selected, the diagnostic measurements, and homeowner preference. The general principles behind the ways to prevent radon from entering the home are to seal the entry routes, to ventilate the soil or space beneath the house, and to prevent depressurization of the house. Once radon enters the home, the concentration must be reduced by increasing the ventilation and effecting dilution. However, increasing ventilation will in turn increase energy costs for some homes because of regional and seasonal weather variations. Each of the general reduction methods can be accomplished in a variety of ways, and at a range of costs. By far the most popular and effective methods are termed active soil ventilation techniques. In employing these techniques, the soil beneath the house is depressurized, which counteracts the seepage of soil gas into the house. For a very high concentration, a combination of methods may be necessary to achieve acceptable radon levels. There are publications that provide information on radon reduction methods for existing structures $(7,10)$. These publications summarize the general principles of radon mitigation and outline the 
design and installation of various reduction methods and the circumstances under which each method is applicable. They also provide estimates of installation and operating costs and the percent reduction that may be expected. There are also publications that describe and recommend construction practices to observe in order to build "radon-resistant" homes $(3,11)$.

\section{A SOLUTION TO THE RADON PROBLEM}

While a vast amount of literature on radon reduction is available, there are relatively few experts around to provide their judgement for each individual who needs a practical and cost effective means to reduce their indoor radon level. Also, as new methods become available, knowledge of them may not be widespread. Radon reduction is a new field, and since the need for it varies regionally, not all contractors have sufficient expertise in dealing with a radon problem (12). In the Pacific Northwest, most of the mitigators are located in the spokane/coeur d'Alene area, where 1 in 3 homes exceed the EPA action level. Some state health agencies have received grants from the EPA for additional monitoring of radon levels, but they are not funded for the mitigation of homes or for the training of mitigators. One solution is to provide the mitigator with a knowledge-based advisory system capable of disseminating the knowledge in the present literature and assisting them in 
various aspects of radon mitigation work.

This thesis describes the development of an advisory system for indoor radon mitigation. The prototype has been developed at Portland State University's Department of Mechanical Engineering under a research grant from Bonneville Power Administration. It is intended to assist Pacific Northwest mitigation contractors in the selection and design of mitigation systems for existing residential homes. The advisory system makes a recommendation as to which mitigation method should be used. In addition, the required computations for a cost analysis and a fan selection are performed. Additional potential benefits of mitigation contractors utilizing the advisory system are verification of approved radon reduction technologies, uniform record keeping for a regional database, and consistency in computational processes. 


\section{KNOWLEDGE-BASED SYSTEMS}

\section{EXPERT SYSTEMS APPLICATIONS}

Knowledge-based systems are categorized as Artificial Intelligence (AI). Some more recent classifications include neural networks and fuzzy logic. In terms of currently available applications, knowledge-based systems have risen to the forefront of AI. Knowledge-based systems applications have increased in the fields of diagnostics, monitoring, planning, trouble shooting, and design. There have been several attempts in the application of knowledgebased systems to building design $(13,14)$, and efforts in applying expert system technology to radon mitigation have also been reported. An initial attempt was made by Mosley in 1987 (15), and a demonstration system on a Macintosh computer was developed by Brambley in 1990 (16). In addition, an interactive system was developed for a Macintosh by Brennan in 1990 (17). The demonstration system illustrated the usefulness of user-directed point-to-point hypertext when working with large amounts of textual information. The interactive system was designed to assist in the training of mitigation contractors. These ventures demonstrated the capability of expert systems in dealing with radon mitigation. 
Expert systems capture the knowledge of human experts in the areas where expertise can be readily obtained and coded via symbolic knowledge processing languages (such as LISP or PROLOG) or expert systems development shells (such as LEVEL5, NEXPERT, OPS83, and others). A knowledge-based expert system is typically comprised of four main components; an inference engine, knowledge base, working knowledge, and user interface. The relation between these elements is shown in Figure 1.

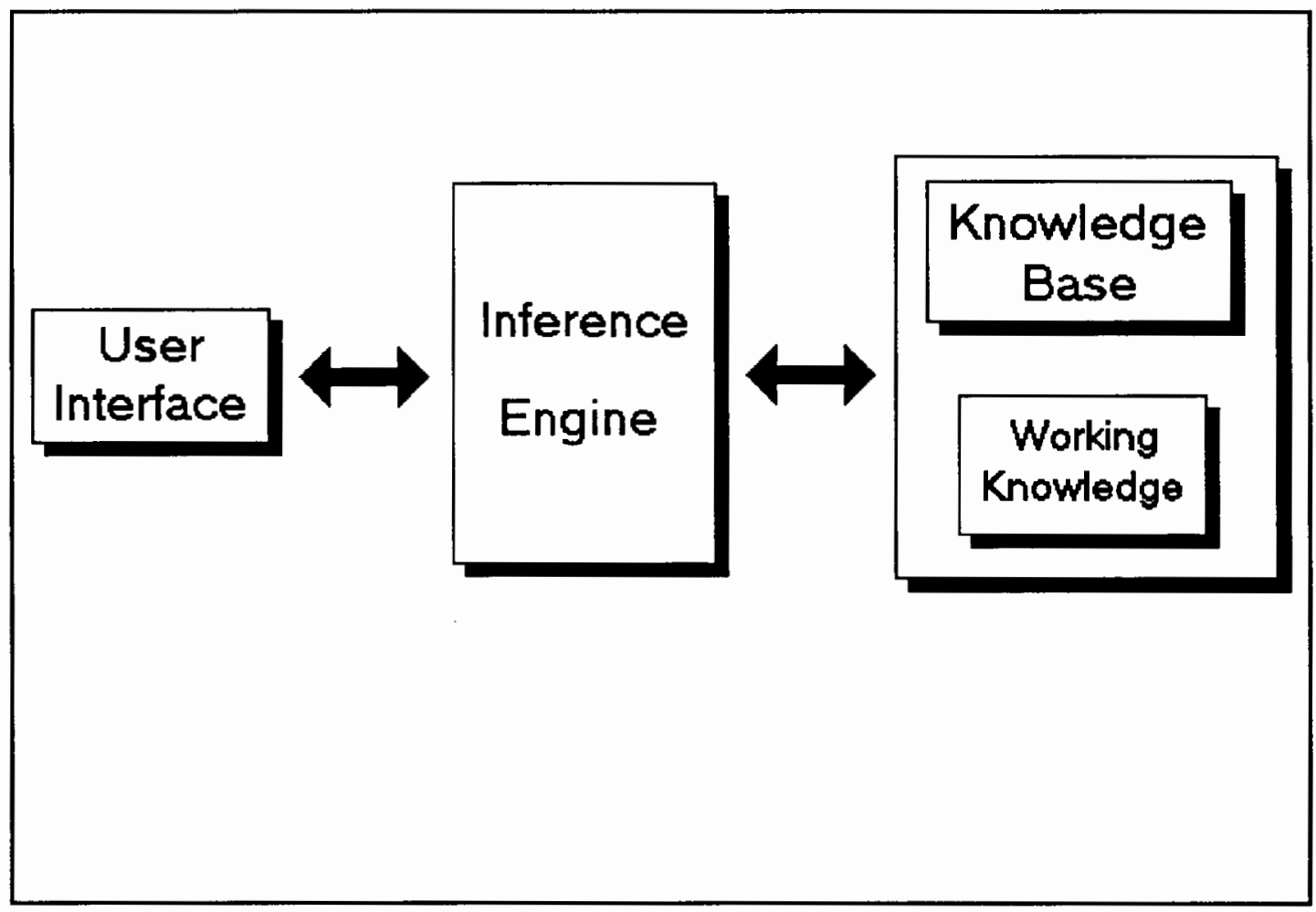

Figure 1. Major components of an expert system. The inference engine implements the problem solving strategy. The knowledge base contains the knowledge 
pertaining to the solution of the problem. The working knowledge represents data relevant to the problem at hand, or case-specific data. This data is extracted from the user through the user interface, which may utilize a variety of techniques for querying the user. Variations of this structure include an explanation facility, natural language interface, and other modules (18). A hybrid system demonstrates an integration of procedural and knowledgebased paradigms (19).

\section{AN OVERVIEW OF LEVEL5}

The computer program has been developed using an expert system shell. Using a shell (versus a pure symbolic processing language) greatly reduces the development time since the inference engine is built-in and can be readily activated without the need for further programing. Another advantage of using a shell is that the user interface can be rapidly customized for the inputs. The particular shell that was used employs a graphical user interface, which enhances the presentation of information to the end user and makes it possible to incorporate graphical as well as textual information.

The work statement for this project required that the advisory system be developed for use on a PC, and take advantage of a graphical user interface. This project started shortly after the release of Microsoft Windows 3.0, 
so it was selected as the development environment. An

illustration of a sample branch of a knowledge tree, shown in Figure 2, shows the advantage of employing a graphical user interface when developing a knowledge base.

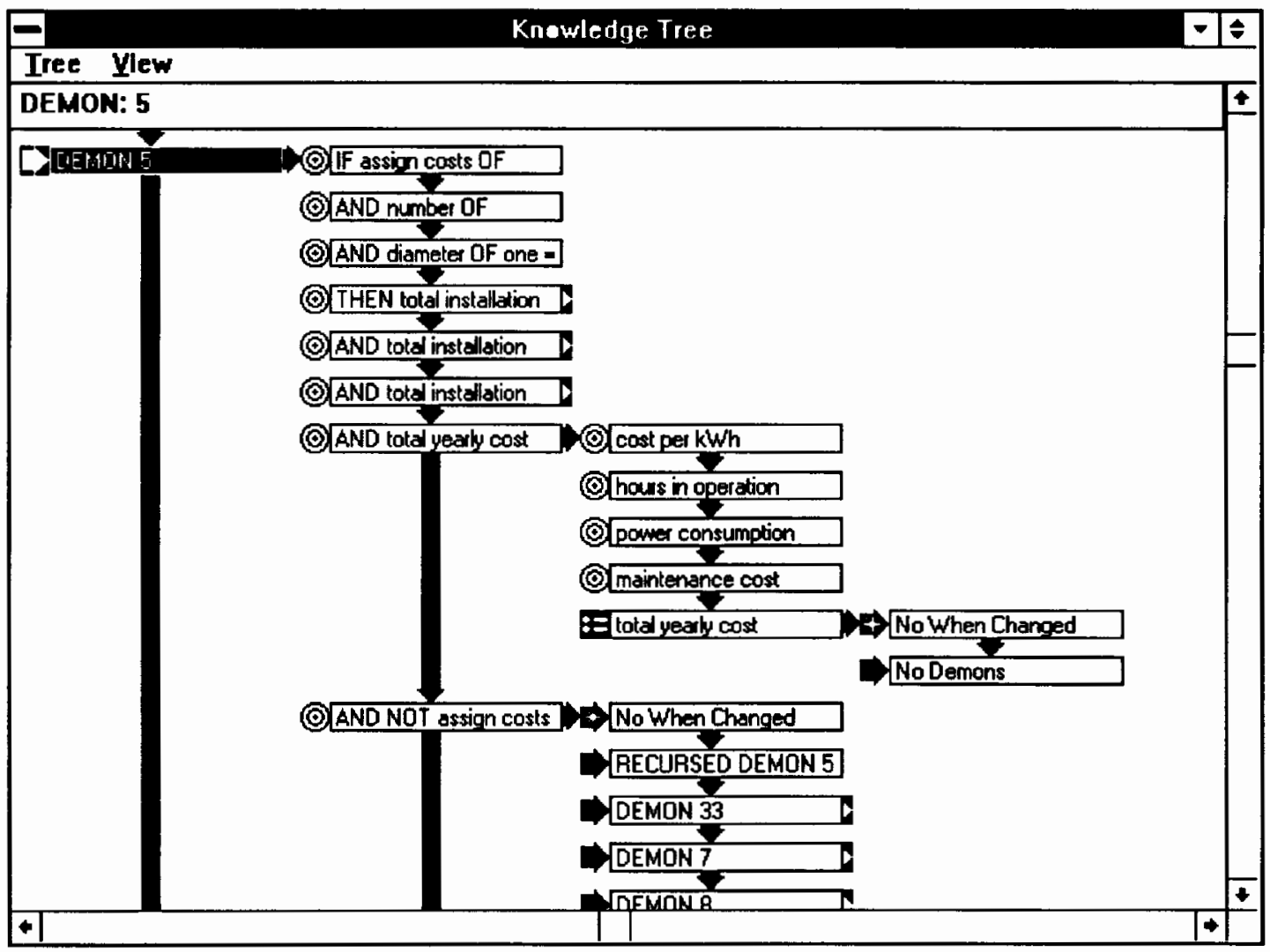

Figure 2. A sample branch of the knowledge tree.

An investigation of the currently available expert system shells that were compatible with windows brought us to LEVEL5 OBJECT from Information Builders, Inc. The shell was readily available at a non-prohibitive cost, and the company offered an educational discount. LEVEL5 OBJECT is an object-oriented software, and supports a multitude of 
inferencing strategies. These include forward and backward chaining, mixed mode (a combination of forward and backward chaining), procedural, object-oriented, and point-to-point hypertext (20). Other features of the software include graphical capabilities, debugging tools and the capability to interface with external programs. One of the features that was explored but not implemented for this phase of the project was the database interfacing capability. There is great potential for this capability, and preliminary reviews of the software indicate that this may be some form of future work on the project.

To create a knowledge base file (.KNB file) using LEVEL5 OBJECT, the required components are the RULES (or DEMONS) and METHODS, the user interface, and the knowledge in object oriented form, that is, arranged as groups of classes, attributes, and instances. A class may be a collection of attributes, and instances are specific occurrences of attributes. The attributes and instances most commonly used are designated simple, compound, multicompound, string, and numerical. Simple attributes may take on values of TRUE or FALSE. Compounds may take on a single value from a list of values. Multicompounds are similar to compounds, but may take on more than one value from a list of values. String and numerical attributes may take on text or number values, respectively. A representation of classes, attributes, and instances is 
shown in Figure 3.

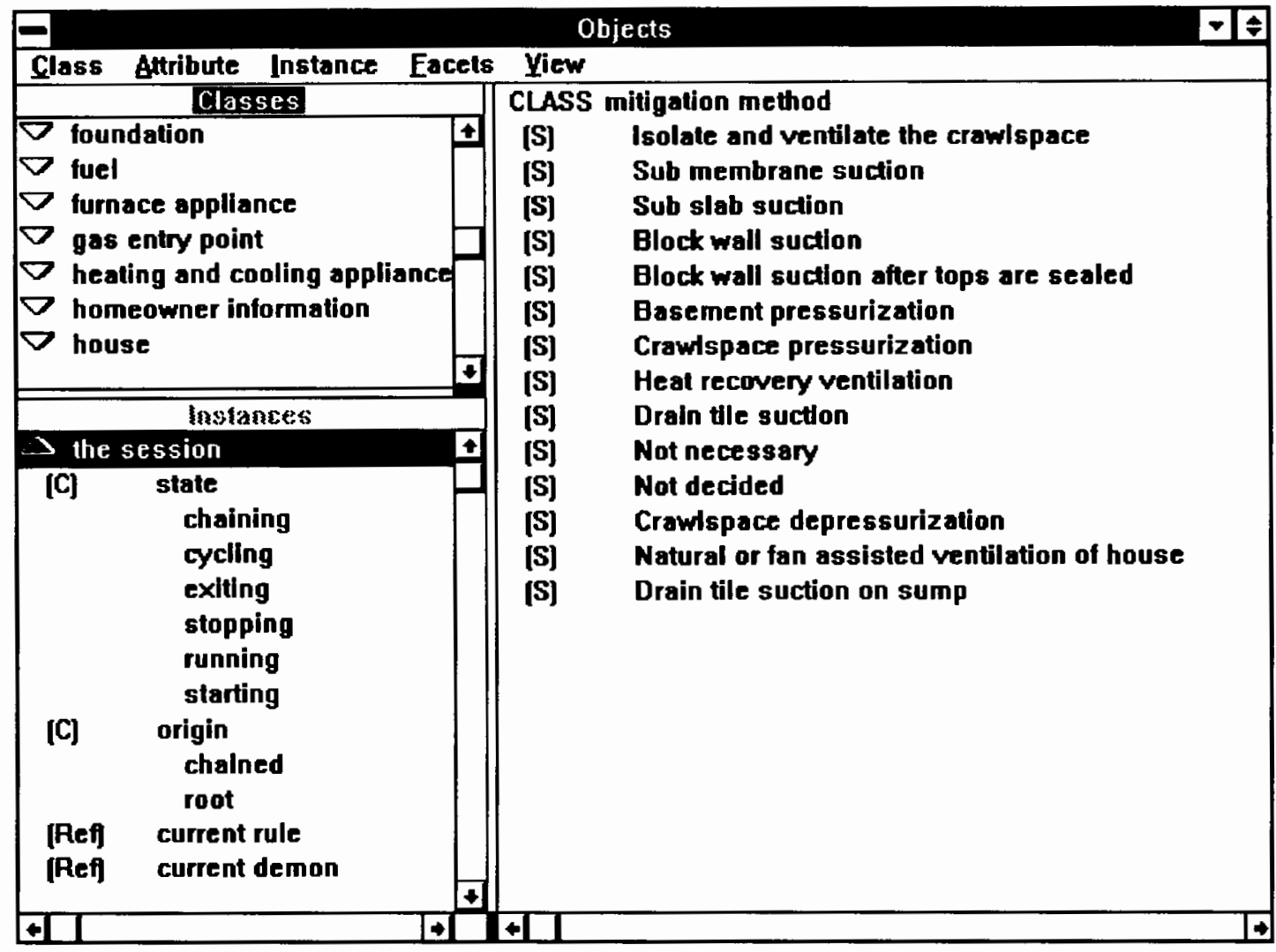

Figure 3. Classes, attributes, and instances.

The user interface is composed of displays (screens) that contain prompts for the user to enter the needed working knowledge or case specific knowledge. The prompts take on different appearances depending upon the attribute type. A simple attribute will offer a choice of TRUE or FALSE. Compounds and multicompounds are attached to graphic tools such as radiobuttons and checkboxes that the user can select with the mouse pointer. Textual and numerical information is entered into promptboxes through the keyboard. An 
example of these tools is shown in Figure 4.

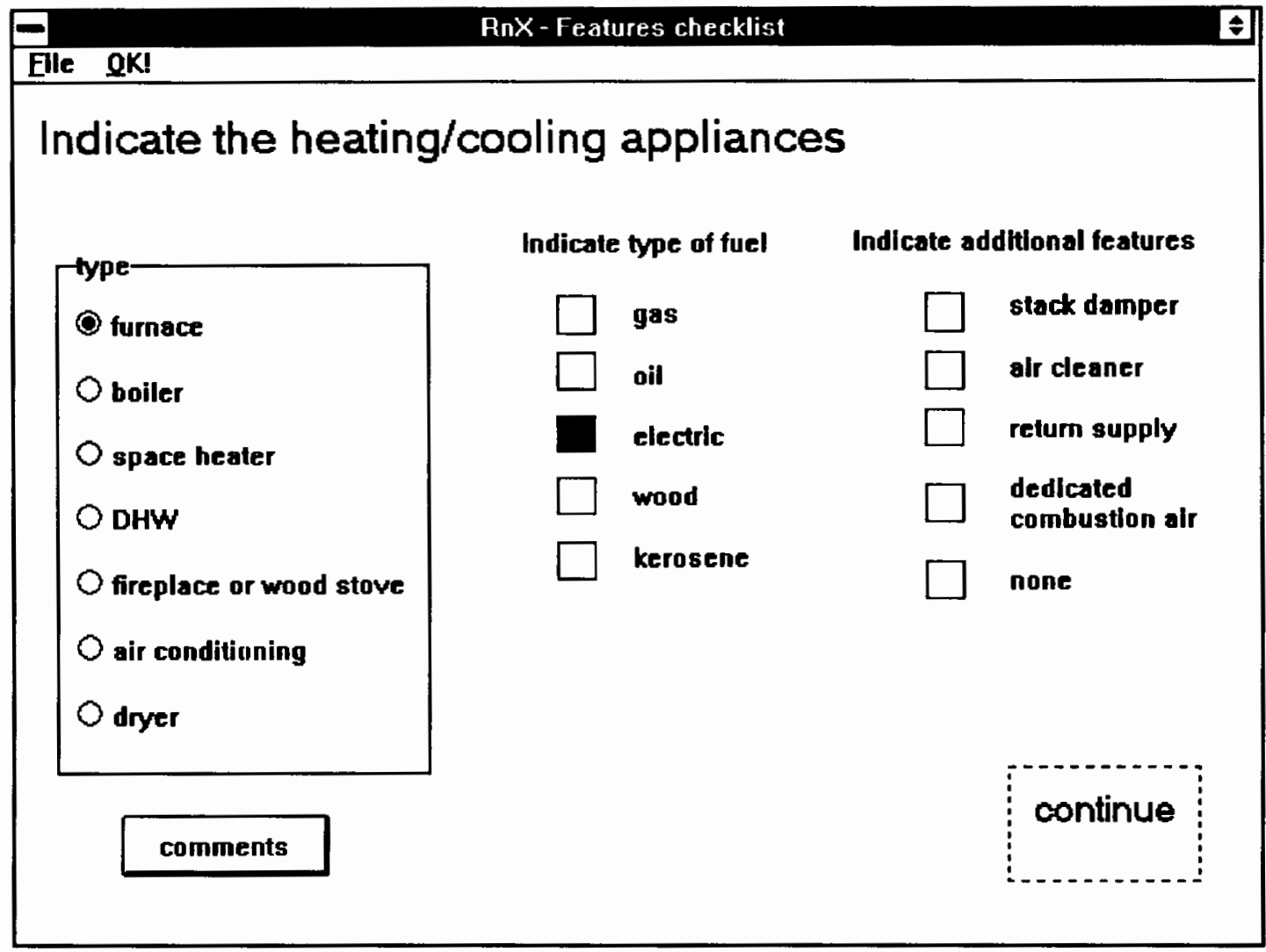

Figure 4. An example of the graphical tools employed.

The LEVEL5 development tool has evolved considerably over the course of this project. At the start of this project, we purchased Microsoft Windows 3.0 along with version 2.0 of the shell. Within several months the shell was replaced by version 2.1. Version 2.0 had encountered problems, and it was later revealed that this version of the shell was intended for operation under a previous version of Windows, and was not fully compatible with windows 3.0 . Several months later, we received version 2.2 of the shell, 
which allegedly featured improved memory management. We have been using version 2.2 of the development tool for the majority of this project. We have found it necessary to operate the windows environment in what is termed as "standard mode" in order to avoid the terminal errors which occur with disturbing frequency in "enhanced mode."

\section{INFERENCING STRATEGIES}

The construction of a rule base in LEVEL5 depends on the type of inferencing strategy that is preferred. DEMONS are employed in forward chaining or data driven strategy, while backward chaining or goal driven strategy uses RULES. Procedural METHODS are employed for both types of inferencing strategies, and are activated when the value of its associated attribute changes during a working session.

Backward chaining or hypothetical reasoning starts with a specific hypothesis (goal) and works backwards attempting to justify the goal. It is best employed in applications where the data is broad or unknown, such as recommendations and medical diagnosis. Forward chaining begins with known conditions, and determines what can be concluded from them. Forward chaining is best used when the data is already available or when the system must respond in real time to changes in information.

For a radon mitigation advisory system, It may seem apparent to use a backward chaining strategy, since the 
system makes a recommendation. However, forward chaining was employed. The reason for this is that the input data is presumed to be readily available, and much of the knowledge obtained from the radon mitigation literature is displayed as flow charts, which are inherently data-driven.

\section{RADON MITIGATION AND KNOWLEDGE-BASED SYSTEMS}

This work details the development of a prototype hybrid knowledge-based advisory system for indoor radon mitigation. The prototype advisory system has been developed employing knowledge obtained from existing publications on radon mitigation. Some additional knowledge has been provided by field experts. Portions of the knowledge are well defined and numerical in nature, such as the fan selection and the accompanying calculations. These portions can be easily implemented in a computerized environment. 
SYSTEM DEVELOPMENT

GENERAL SYSTEM STRUCTURE

The advisory system, named RnX, is composed of six modules. A modular structure was adopted to limit the size of the separate components, and to facilitate debugging and potential expansion of the system. Each module is composed of its own DEMONS, METHODS, classes, attributes, and instances. A listing of some of these items is included in the appendices.

Some of the modules use the same classes and attributes. The values of these attributes are obtained from the user by one of the modules (detailed later in this chapter), and passed between the modules during execution. The method used to pass the data was to write the information to a text file which in turn is accessed by a subsequent module.

The House Investigation Summary module (RNXI.KNB) extracts the characteristics of the house. This module was designed to be analogous to the house investigation summaries that mitigators often use to gather pertinent data about a particular house $(7,8,10)$. The Data Conversion and Mitigation selection module ( $\mathrm{RNX2}$.KNB) converts the data from the previous module into a form that is usable by the 
system, processes its rule base, and recommends an appropriate mitigation method. These first two modules are the most essential ones, and constitute the mitigation determination process. The Suction Point Determination module (PNT.KNB) assists in the point selection (the number of needed suction points) for soil suction techniques, the Fan Selection module (FAN.KNB) performs a ducting analysis and fan selection for sub-slab suction techniques, and the fifth module performs a cost estimation (COST.KNB). The modules are activated hierarchically, as shown in Figure 5 .

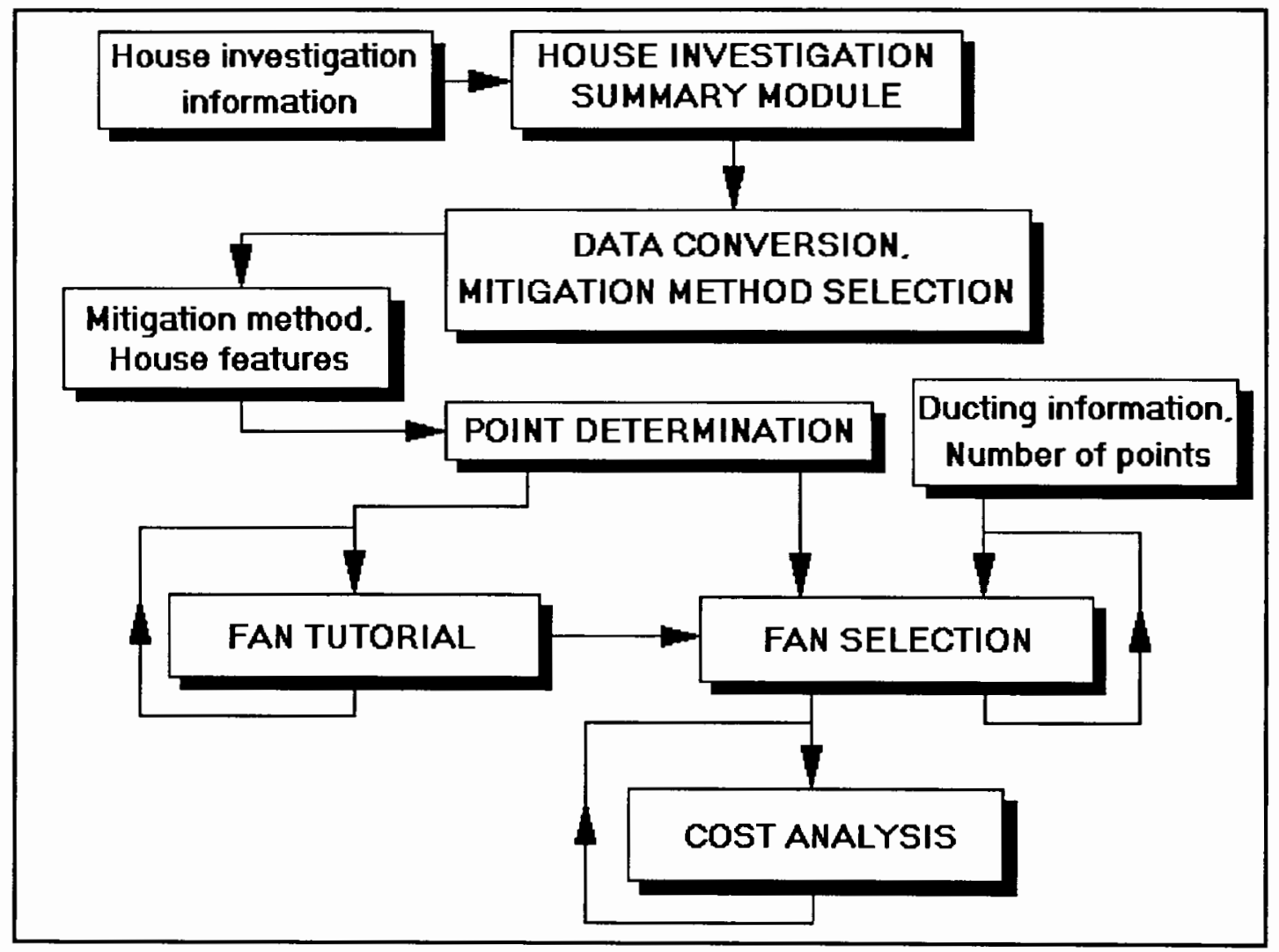

Figure 5. A representation of the system modules. 
Once the mitigation determination modules have been executed for a particular case, subsequent modules such as the Fan Selection or the Cost Estimation can be repeatedly executed without invoking the previous modules. This arrangement makes it possible for the user to reactivate individual modules as many times as is desired. An additional and completely independent module is the Fan Tutorial, (FANTUTOR.KNB) which is similar to the Fan selection module. This module provides on-line assistance and defines the notation that is used for the Fan selection module. In the prototype, some modules provide an on-line utility for the software reviewers to make comments and suggestions for each step of the system. This utility is to be employed for the testing phase of the software as a method to collect a wide spectrum of expert opinion and enhance the robustness of the system.

The hybrid nature of the entire system is evident in its integration of heuristic and procedural methods. The heuristic portions of the rule base pertain mostly to the mitigation method selection. The procedural methods are exclusively for the ducting analysis, fan selection, and cost estimation.

HOUSE INVESTIGATION SUMMARY

In the House Investigation Summary module of the advisory system, the user is queried about the 
characteristics of the house. The information sought by the module is identical to that requested by house investigation summaries used by professional mitigators. An advantage to this approach is that the mitigator can fill out house investigation forms while in the field, and can use the system at a later time. The queries on the forms were grouped into classes and attributes. For example, sub-slab material may be specified as gravel, clay, loam, sand, or unknown. Sub-slab material would be classified as a compound attribute, where one and only one of the choices may be selected. Some other attributes include the percent composition of the foundation (numerical attribute), the kinds of thermal bypasses present in the house (multicompound attribute, since more than one bypass may be present), and the presence or absence of air ducting (simple attribute). The queries start out as very general and evolve to ask for greater detail. In this way, only relevant questions will be asked. For instance, if in response to the query on foundation type a house is specified as having a full basement, the system will continue to seek out more information about the basement, and there will not be any questions asked about crawlspace features. This illustrates the data-driven strategy of the system.

The working knowledge extracted from the user is written to a user-named text file which is referred to by 
the Data Conversion and Mitigation selection module (see Figure 6).

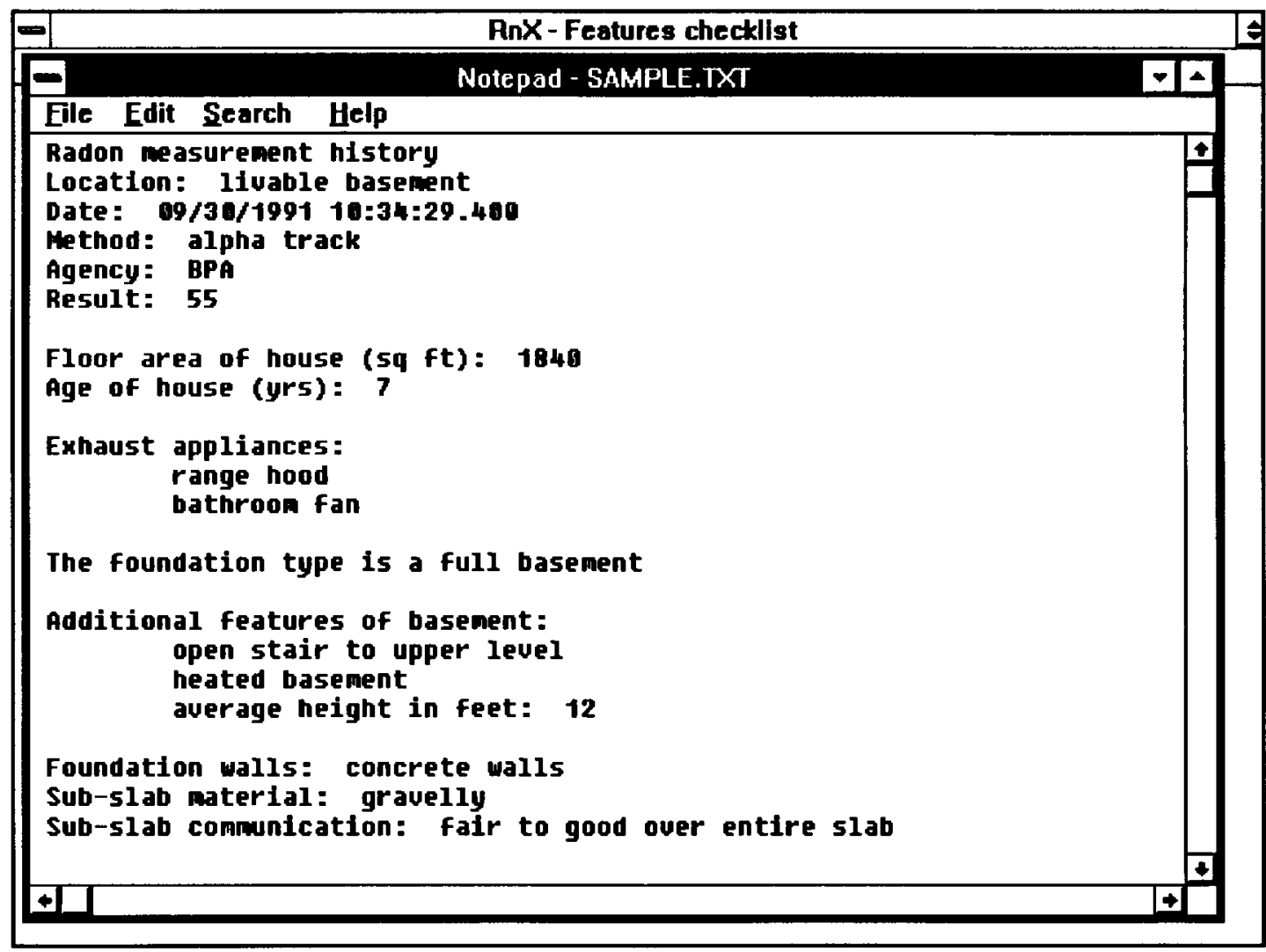

Figure 6. A portion of the working knowledge.

That module utilizes this file and applies the inferencing strategy to the present problem context. This file can be accessed by the user for viewing and some on-line editing, but a strict format must be followed in order to maintain its compatibility with the following module. For major changes, it is recommended that the user rerun the module and create a new text file.

The module employs point-to-point hypertext in 
conjunction with METHODS and DEMONS to link the displays and control the flow of the queries. The module begins by prompting for the name and address of the client. This information is stored in the first few lines of the data file, and will be displayed by the following module prior to DEMON processing to allow the user to confirm that the proper file will be used. The flow of this module is dictated in part by the user input. For example, when the user inputs the number of radon measurements that have been conducted, the system will respond with additional promptings (location, measurement method, etc.) for each of those measurements. The location of the radon measurements were generalized to five areas; crawlspace, livable basement, non-livable basement, frequent living area, and infrequent living area. The location of a radon measurement assists in determining where the radon problem is most severe. The date of the measurement is recorded, as this information may help in determining the accuracy of the measurement. Summer measurements are typically much lower than winter measurements due to increased ventilation (open windows), although testing protocol calls for the house to be closed as much as possible during a test. succeeding queries include site characteristics (land, water and climate), the age and square footage of the house, percentages of above grade construction, percentages of interior and exterior finish, the type of heating/cooling 
and combustion appliances, thermal bypasses, foundation type, foundation wall type, percent finish of the foundation floor and walls, types of drainage systems, floor penetrations, sub-slab material, and the degree of sub-slab communication.

Not all of the information gathered by the module is used in the determination of the mitigation method. Some of the queries, such as land characteristics and house age, were included to enhance the record-keeping capability of the system. Others, such as the percentages of the interior and exterior finish, are included but not used in the mitigation determination because there is not any published information on how those items affect a radon problem and how knowledge of them would influence the selection of a mitigation method.

The module informs the user when it has completed its queries, and presents the user with an opportunity to inspect the newly generated text file containing the working knowledge.

DATA CONVERSION AND MITIGATION METHOD SELECTION

The Data Conversion and Mitigation Selection module reads the text file created by the House Investigation Summary module and assigns appropriate values to the objects of the knowledge base. The text file is read and converted by using several METHODS. This module is similar to the 
previous module in that the objects of the knowledge base correspond to a house investigation summary. However, this module does not require as many displays, employs much less point-to-point hypertext, and has a vastly different rule base. The DEMONS and METHODS of the system are based upon information contained in radon mitigation literature, and are listed in the appendices. DEMON processing is activated only after all of the data has been converted into objects. Additional information not included with the house investigation summary, such as the accessibility of the crawlspace, may be requested to complete DEMON processing. An example of one DEMON is :

\section{DEMON SAMPLE}

IF radon concentration $>20 \mathrm{pCi} / \mathrm{L}$

AND foundation OF house IS basement

AND type OF foundation wall Is poured concrete

AND degree OF sub-slab communication Is good

THEN recommended mitigation method Is sub-slab suction

The antecedents of the DEMONS were structured to fail as early as possible. That is, the most general statements were placed before the detailed ones. In the DEMON shown above, the first statement concerns the concentration. This information determines whether or not a radon problem even exists. The following statement, concerning the foundation type, will determine which mitigation methods are applicable. Statements following the foundation type are usually specific to that foundation type. A mitigation method is recommended only after conditions for its 
applicability are met and DEMON processing is completed (see Figure 7).

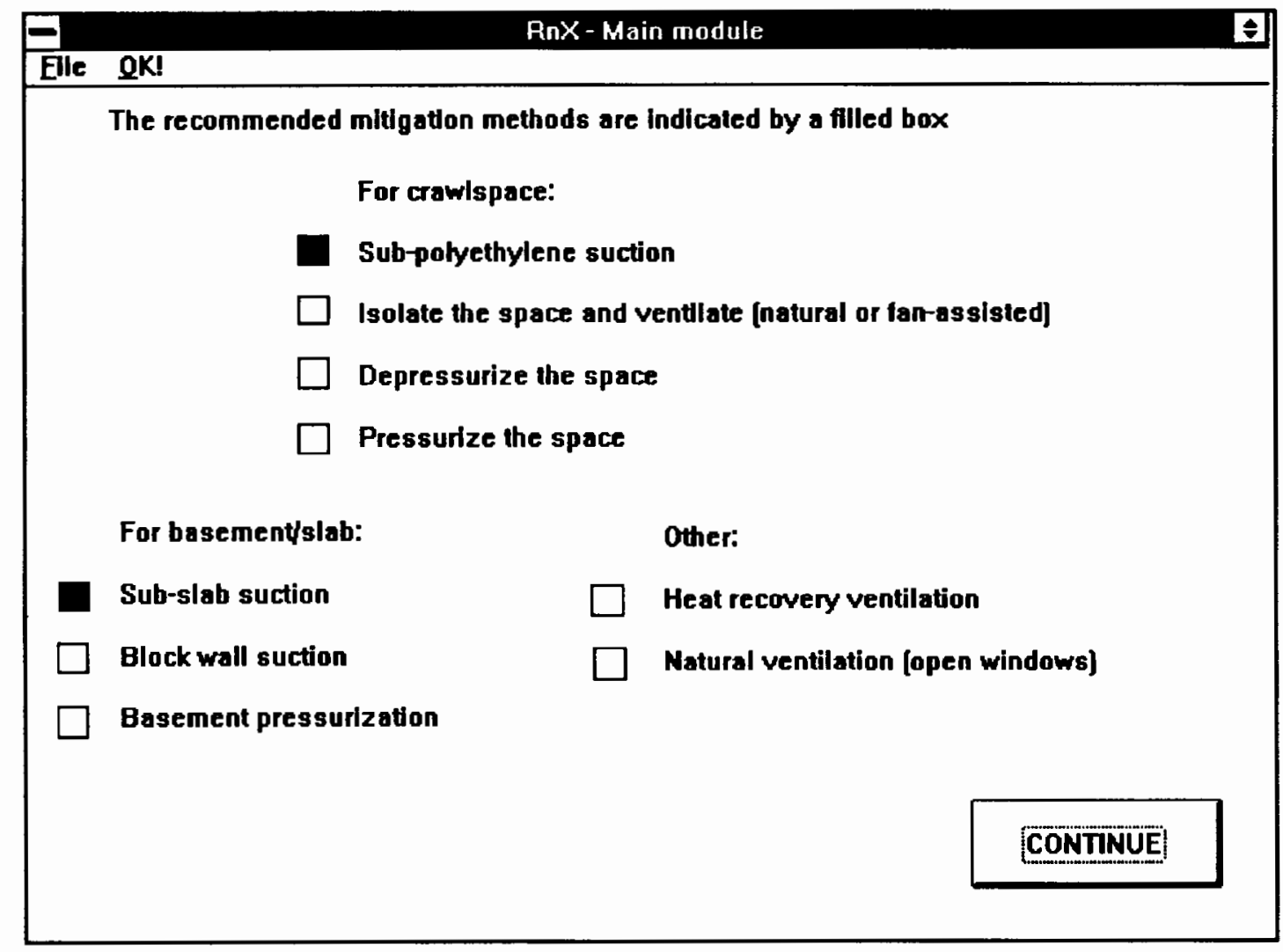

Figure 7. The recommended mitigation methods.

More than one mitigation method may be recommended, especially in the case of a foundation consisting of a combination of simple foundation types, such as a basement adjoined to a crawlspace. At this point, the user may select one of the recommended methods based upon an intangible factor, such as aesthetics. If all relevant data has been collected and processed, and the module fails to find a method, the user is notified of the condition. The 
user is also informed of which floor penetrations were indicated from the house investigation summary and need to be sealed prior to beginning mitigation work. The user is also reminded of appliances that are present and may be contributing to house depressurization. If any of the recommended mitigation methods included a soil suction technique, the user may continue with the suction Point Determination module.

\section{SUCTION POINT DETERMINATION}

The advisory system is capable of recommending the number of suction points that should be installed for soil suction techniques. This recommendation is based on data from a radon mitigation publication (21). The data in this publication is based upon the type of mitigation method that is implemented, the area of contact between the slab and the soil, and the degree of soil communication beneath the slab. Studies have shown that for sub-membrane suction mitigation methods employed in crawlspaces, a single suction point is adequate (22). However, for sub-slab suction mitigation methods, the number of points must take into account the degree of soil communication. If the degree of communication is poor, then the number of points must take into account the square footage of the slab. The present literature fails to agree on a value for the number of square feet per suction point. The values range from 600 
square feet to 1000 square feet per suction point. Since the module bases the number of suction points on the square footage of the slab only if the soil communication is poor, the most conservative value of 600 square feet per suction point is used. If good communication exists, two suction points will be adequate for a typical slab as shown in Figure 8 .

Elle QQK!

The system recommended Sub slab suction and the communication was determined to be good (coarse aggregate). For a typical slab, two suction points are usually sufficient.

Click here to continue

Figure 8. Suction point recommendation.

For block wall suction techniques, the number of suction points corresponds directly to the number of walls that are determined to have elevated radon levels (major walls). The 
module obtains most of its needed information (mitigation method and degree of soil communication) from the previous module. If additional information is needed, such as the area of the slab or the number of major walls, a suitable prompt is presented to the user. At the conclusion of the module, the user can CHAIN to the next module. One future enhancement of the suction point determination module will be to obtain the data concerning the slab area from the previous module, since the total square footage of the house as well as the percent composition of the foundation floor is part of the working knowledge. In addition, the number of partitions created by the presence of footings beneath the slab will have to be taken into account, since the footings will disrupt the extension of the pressure field under the slab. Other future work may include the incorporation of a recent study conducted at Lawrence Berkeley Laboratory on the extent of the sub-slab pressure field extension based upon the relationship between the degree of soil communication and the nature of the sub slab material.

\section{FAN SELECTION}

The purpose of the fan selection module is to perform a piping analysis and to specify the requirements of a fan that is to serve in a sub-slab suction technique for radon mitigation. Appropriate fan requirements can be determined 
only after the flow and pressure requirements of the system (diagnostic measurements) are determined by the mitigator. The module is currently limited to systems with three or less ducting branches (suction points), and duct diameters of 3,4 , or 6 inches. The point restriction was imposed to limit the size of the rule base, and because it is considered common practice to use several smaller fans instead of one large fan in mitigation systems that require many suction points. For example, a system with five suction points would probably have three points going to one fan and the remaining two going to an additional fan. A large fan naturally consumes more power, and makes more noise. Oversizing of fans may lead to increased heating costs for the homeowner due to the year-round operation, and it may precipitate a potentially fatal situation by inducing the back-drafting of combustion appliances. The duct diameters were selected as the most frequently used and readily available sizes.

This module requires that the user have diagnostic measurements available and a preconception as to how the ducting will be configured (23). If not, the user can invoke a fan tutorial module, which is described later. The system passes the data obtained pertaining to the number of needed suction points (determined from the previous module), and the user is given the option of overriding the information and entering a different number. The number of 
suction points corresponds directly to the number of branches in the ducting system. The module requests information concerning the ducting, such as the diameter, length, and the number of fittings used (see Figure 9).

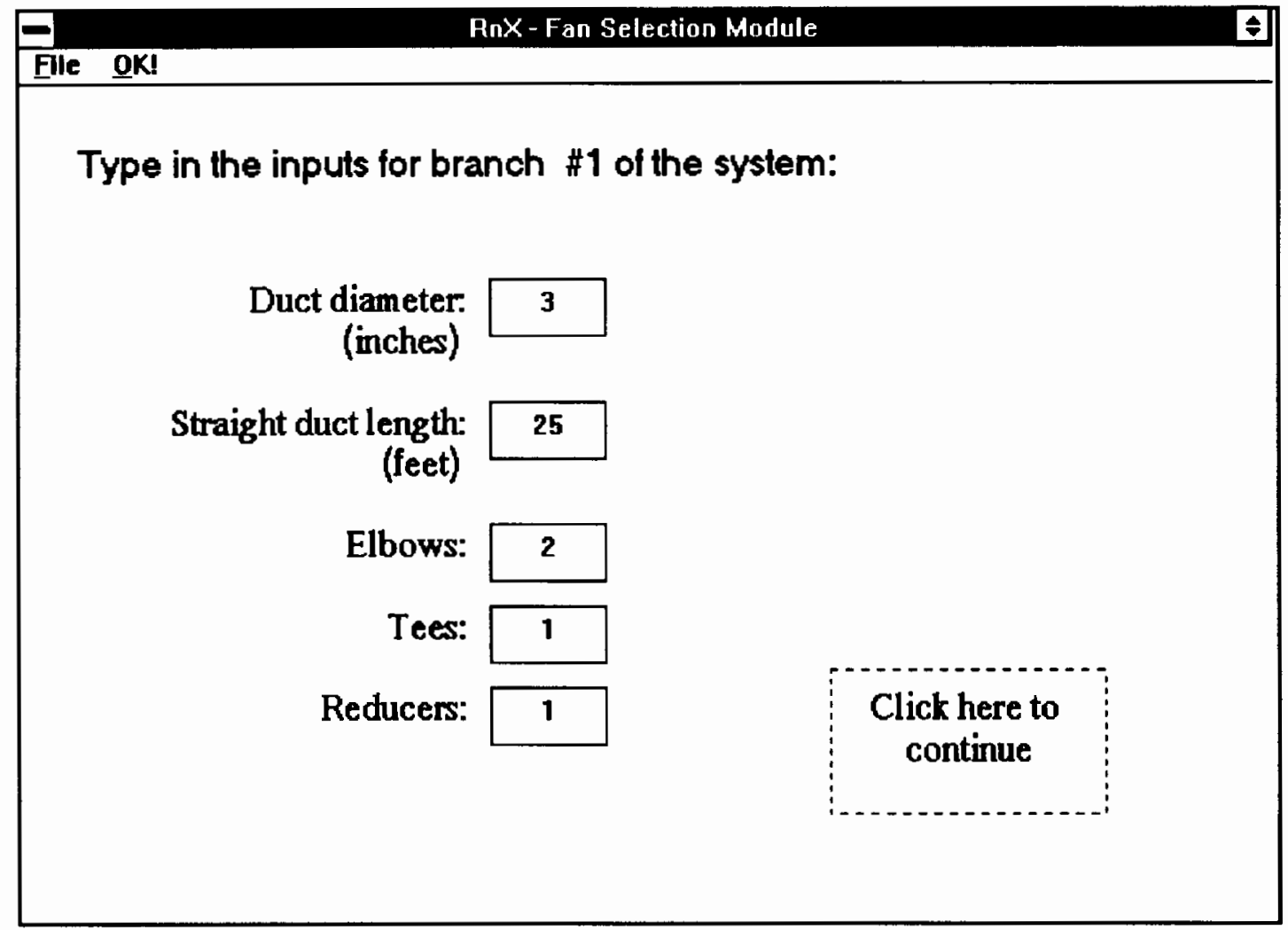

Fiqure 9. Data requested for ducting branches.

For single branch systems, the module assumes that the diameter of the ducting remains constant and that no reducer/expander type fittings are used. For multiple branch systems, different diameters may be used for the different branches. Usually the ducting that makes up the main trunk of the system has a larger diameter than the ducting of the branches, in order to accommodate their 
combined flow without increasing the velocity. For threebranch systems, the module assumes that branches one and two form an intermediate junction, and this intermediate branch joins with the third branch to form the main trunk which goes to the fan. Diagnostic measurements of pressure drop and flow rate are also requested from the user (see Figure 10).

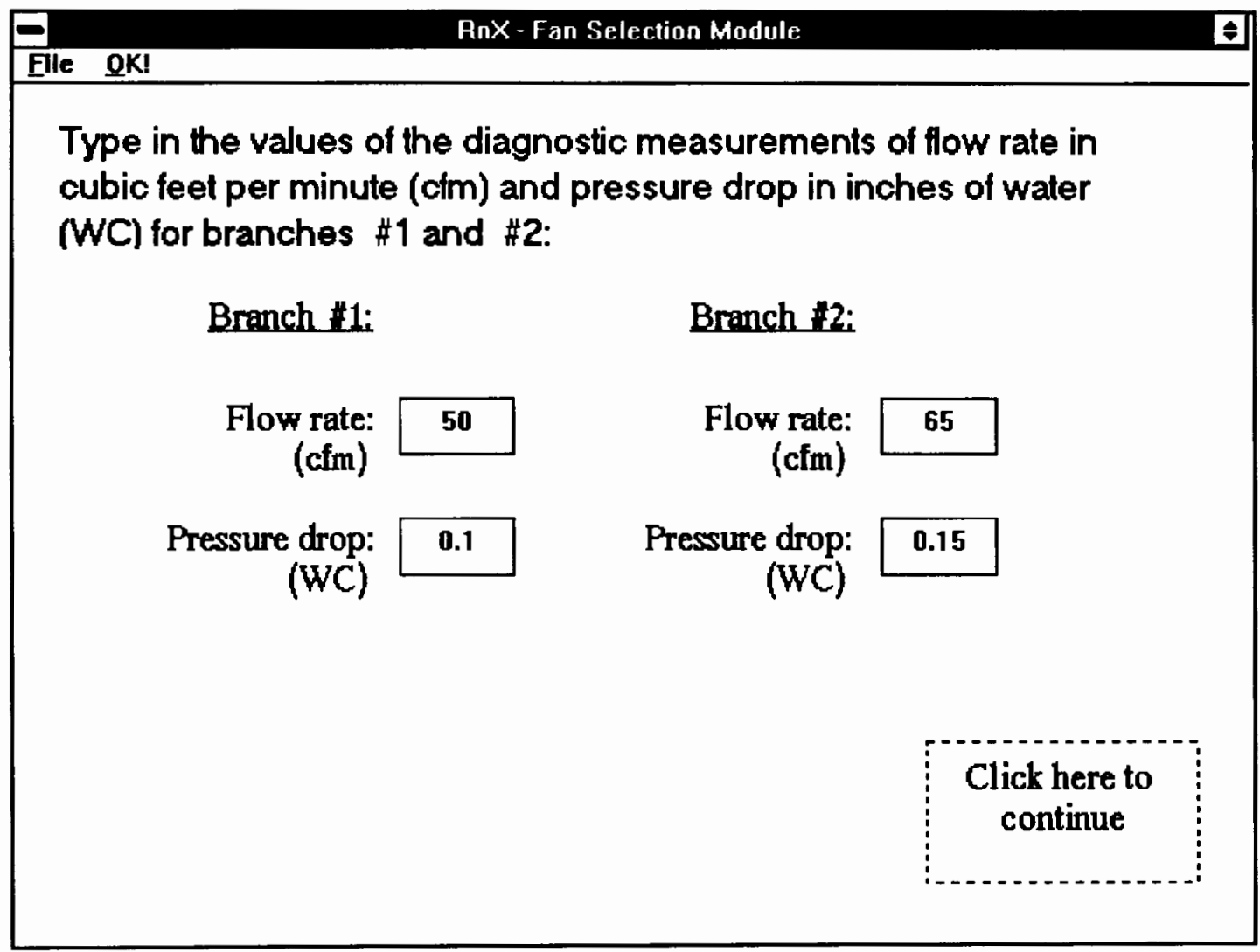

Figure 10. Diagnostic measurements requested.

The module proceeds to determine the total system friction loss and airflow. It uses this data to recommend a generic fan in terms of pressure drop and flow rate as shown in 
Figure 11 .

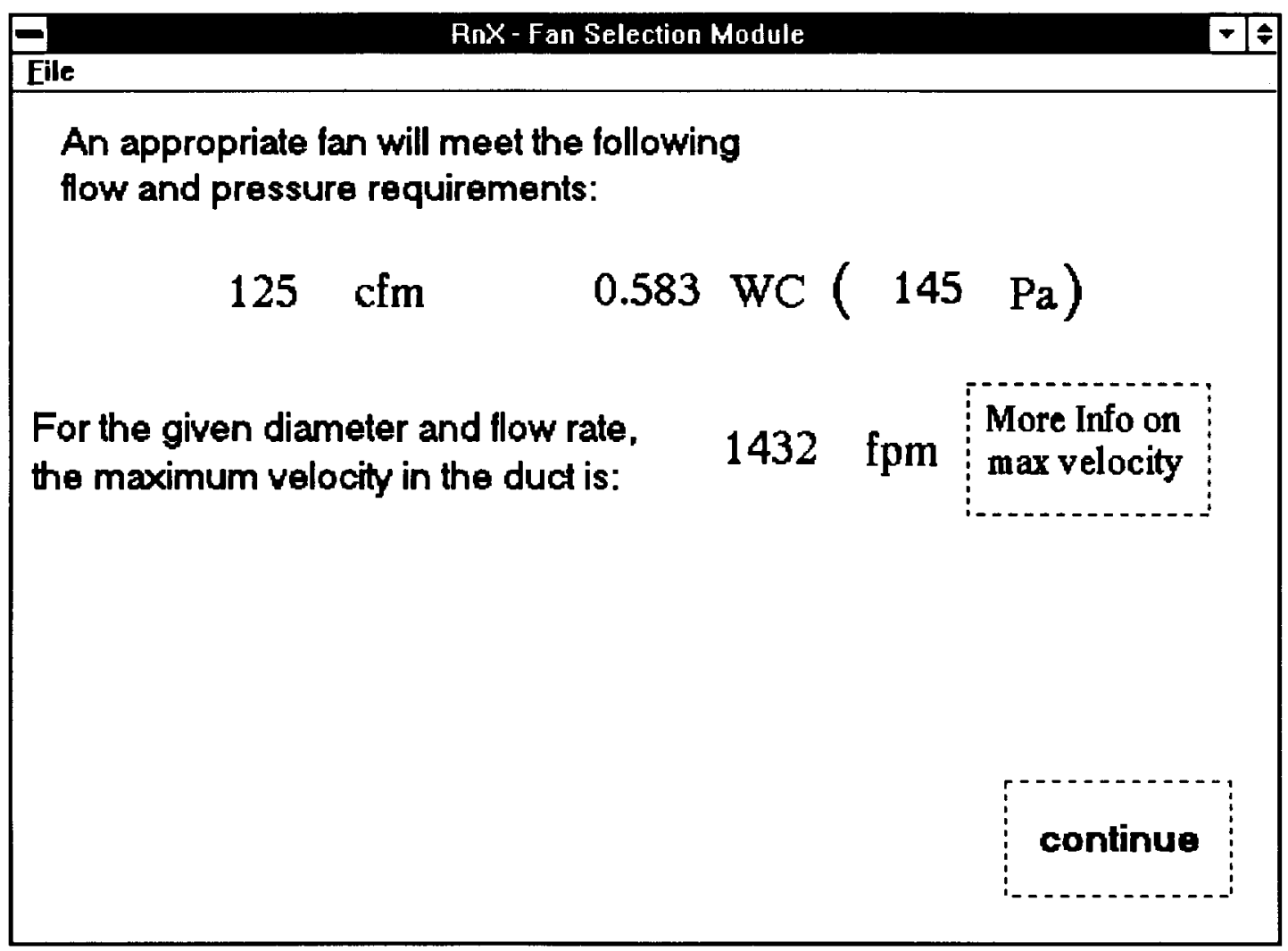

Figure 11. Generic fan requirements.

Once the user is presented with the flow and pressure requirements of the specified ducting system, the user may then wish to consult fan performance charts to select a fan. Maximum air velocity is provided to inform the user of a potential noise problem. A subsequent screen informs the user which branch in the ducting system possesses the highest velocity. This information is furnished to the user in order to facilitate any needed revision of the ducting system. 
The process of the fan selection is very simple if only one suction point is present. When there is only one branch, the diameter of the ducting will be constant, and the only fittings used along the ducting will be elbows. A tee joint may be placed along the ducting to accommodate future expansion to an additional suction point, but it would be sealed on one side and would function as an elbow. The process becomes more complex if multiple suction points are needed to create an effective pressure field beneath a concrete slab. The complexity increases even more if the flow and pressure requirements are different for each branch, which would be the case where sub-slab communication is good at one suction point and poor at another point. If the airflow requirements are vastly different, then the diameter of the branches may vary. A suction point that does not require a high flow rate will not need a large diameter duct even though it may need to have a high suction drawn upon it. A branch with a higher flow may need a larger diameter to reduce the noise caused by the higher velocity flow.

Friction losses along a duct are usually determined by consulting an ASHRAE chart showing a family of curves for duct diameter and velocity (24). The $x$ - and $y$-axis of the chart show friction loss in inches of water (WC), and flow rate in cubic feet per minute (cfm). The friction loss along the length of the duct for the individual branches and 
for intermediate branches and the trunk formed by the joining of multiple branches must be determined. For multiple suction points, the chart is typically consulted several times because the diameter of any intermediate branches and the trunk may be larger to accommodate the combined airflows.

To select an appropriate fan, a fan performance chart must be consulted. These charts plot pressure versus flow. If the point where the required flow and pressure meet is far beyond the curve, the fan is undersized. If the point is far below the curve, the fan is oversized. An important note is that the pressure and flow requirements are usually not specified as a point, but as ranges, for example, 250 to $300 \mathrm{cfm}$ and 1.0 to 1.2 WC. The current version of the Fan Selection module does not use ranges to specify the fan. The Fan Selection Module begins by asking the user for the number of suction points. The response to this will determine the next display sent to the screen. Several different displays were created, one for each case (one point, two points, three points). For the case of a single point, the user is prompted for information about the ducting, which includes duct diameter, the length of the straight portions of the ducting, and the number of fittings used. The straight length and the fittings are needed to determine the equivalent length of the ducting for friction loss purposes. Equivalent length is determined by 
multiplying the number of each type of fitting by an appropriate factor (for example, each elbow is equivalent to 10 feet of length), and adding this to the total straight length. The user also must know the pressure and flow requirements of the system at the soil. These are the diagnostic measurements, which must be recorded at the site. After this information is entered, the user may continue or exit the session. If the user chooses to continue, a simple attribute with a default value of FALSE is assigned a value of TRUE. After the user closes the display, the knowledge base fires DEMONS based on whether or not the IF portions evaluate to TRUE. In this module, the first DEMON to fire after closing the display is one that will calculate the equivalent length of the ducting (DEMON 4). This same DEMON sets another simple attribute to TRUE. This additional attribute is referred to in the IF portions of following DEMONS, since equivalent length must be used in subsequent calculations (DEMONS $5-7$ ). These calculations determine the friction losses along the duct. As previously mentioned, the customary procedure is examine an ASHRAE chart, but the system cannot read the chart, and the user may not know how to read the chart. Fortunately, the chart is a log-log graph and the family of curves is actually a family of straight lines. The duct loss can be determined by a simple equation. As an example, for a 3 inch duct (similarly for 4 or 6 inch ducts), the equation for duct 
loss in terms of flow rate is:

$$
\text { duct loss }=10^{1.85 *(\log (\text { flowrate })-1.76)}
$$

The chart gives the duct loss per 100 feet of ducting, so the result is adjusted to correspond to the equivalent length. After the calculation is completed, another simple attribute is set to TRUE, which is used as part of the antecedent of a later DEMON, which will assign values to the total flow rate and the total system friction loss. In the case of one suction point, the total flow rate is the diagnostic flow rate. The total system friction loss is the sum of the diagnostic friction loss measured at the suction point and the duct loss that occurs due to friction along the length of the ducting. The total flow rate and total system friction loss are used to select the appropriate fan (DEMONS $40-46$ ).

For the case of two branches, the user must enter the duct information and the diagnostic measurements for both branches. Additional fittings are included, such as tees and reducers. Furthermore, the user must enter duct information for the trunk that is formed by the joining of the two branches. The system assigns the flow rate for the trunk by summing the flows of the branches. The duct loss for the trunk must be calculated separately, since the trunk diameter will probably be larger than either of the branch diameters. The total system friction loss takes into account the diagnostic friction losses at the soil and the 
three duct losses (two branches plus one trunk).

For three branches, the procedure is similar to that of two branches. The user enters the diagnostics and the duct information for the three branches, and then needs to enter the duct information for the trunk formed by two of the three branches. This first trunk and the remaining branch will form a second trunk. The second trunk has to be analyzed before the fan is selected, because the total system friction loss is the sum of the diagnostic measurement, the branch duct losses, and the trunk duct losses.

One problem encountered during the development of this module was the initial value settings of the numerical attributes. One of the attributes used in a calculation was never prompted from the user. This attribute was not the result of a calculation, so the only way for it to be assigned a value was from the user. This attribute retained a value of UNDETERMINED. This value propagated from the original equation containing the attribute, all the way through to the end of the knowledge base session. This problem was solved by setting all of the initial values to zero. However, this caused the knowledge base to test all of the DEMONS before displaying the title screen. The problem was resolved by changing all of the initial values back to UNDETERMINED and prompting the original problem attribute from the user. 
A future enhancement of this module is to incorporate some way to select the fan when the total flow rate and total system friction loss are given as a range instead of a point. Additional fittings could be included to analyze exotic duct configurations, and, of course, the system could stand to be expanded to handle multiple suction points - any number that the user specifies, within reason.

The module also shows the maximum air velocity achieved, and in which branch of the system it occurs (see Figure 12).

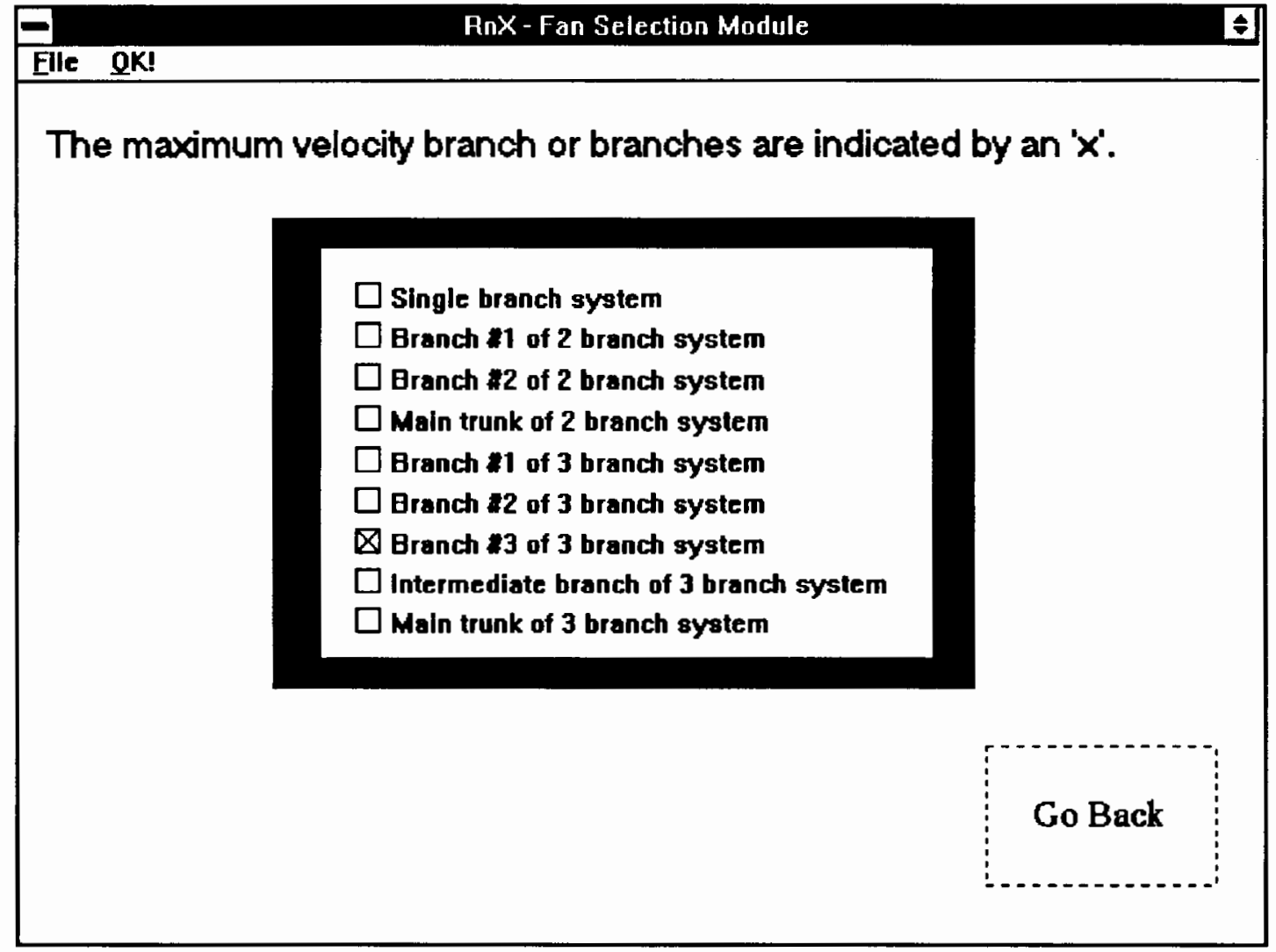

Figure 12. Information on duct velocity. 
The information on air velocity is provided in order to notify the user in the case of a high velocity flow. This makes the user aware of a potential noise problem and identifies the portion of the system that may need to be redesigned. The Fan selection module may be repeatedly executed to permit the user to experiment with the design of the ducting system. For instance, if a high velocity value is detected in a branch with a three inch diameter duct, and the value is perceived by the user to be capable of causing excessive noise, the system can be redesigned using four inch diameter or larger ducting. This process can be repeated until the user is satisfied with the design. A future enhancement may include having the module make the determination as to the occurrence of noise-causing flow, instead of having the user make this determination.

Furthermore, the cost Estimation module can be invoked to determine the impact on the total system cost due to changes in system design. Also, once the user has received the generic fan requirements, but does not wish to consult fan performance charts to select a fan, a small selection of some brand name fans, and the models that will meet the performance requirements are presented (see Figure 13). Additional information, such as the listed purchase price and the power consumption of the fan, is provided to assist the user in selecting a fan to be used with the cost estimation. 


\begin{tabular}{|c|c|c|c|}
\hline \multicolumn{4}{|c|}{ AnX-Fan Selection Module } \\
\hline \multicolumn{4}{|c|}{ Wattage } \\
\hline Kanafflakt & K6 & 120 & $\$ 180.00$ \\
\hline Rosenburg & $\mathrm{R} 200$ & 125 & $\$ 138.50$ \\
\hline Fantech & $\mathrm{F} 200$ & 100 & $\$ 160.00$ \\
\hline Vent-axia & Powerfan ACP150 & 85 & $\$ 110.00$ \\
\hline Powerfan & & & \\
\hline \multicolumn{4}{|c|}{$\begin{array}{l}\text { This is a list of some brand name lans. If you have } \\
\text { a preference for one of the listed fan brands, position } \\
\text { the mouse pointer over it and click once. The cost } \\
\text { and energy consumption will be used in the cost } \\
\text { estimation. If you do not have a preference, the } \\
\text { system will select the least expensive brand. }\end{array}$} \\
\hline
\end{tabular}

Figure 13. Brand fans capable of meeting requirement.

\section{COST ESTIMATION}

The purpose of the cost estimation module is to provide the user with a realistic estimate of the costs involved in the installation, operation, and maintenance of a mitigation system. The cost estimation module sums up the material cost for the ducting and the type of fan specified in the fan selection module, and adds it to a labor cost, an energy cost, and a miscellaneous cost. The result is an estimation of the total cost for installation of the mitigation system and the annual cost for operation and upkeep of the system. Attributes passed from the fan selection module include the 
selected fan along with its cost and power consumption, the lengths and diameters of the ducting and the number of each type of fitting used. The values for these attributes are applied to default unit costs. The purchase costs and power consumptions of the fans were obtained from specifications provided by the manufacturer or dealer of the fan. The default values for the ducting costs were obtained as offthe-shelf prices from a local home improvement store (see Figure 14) .

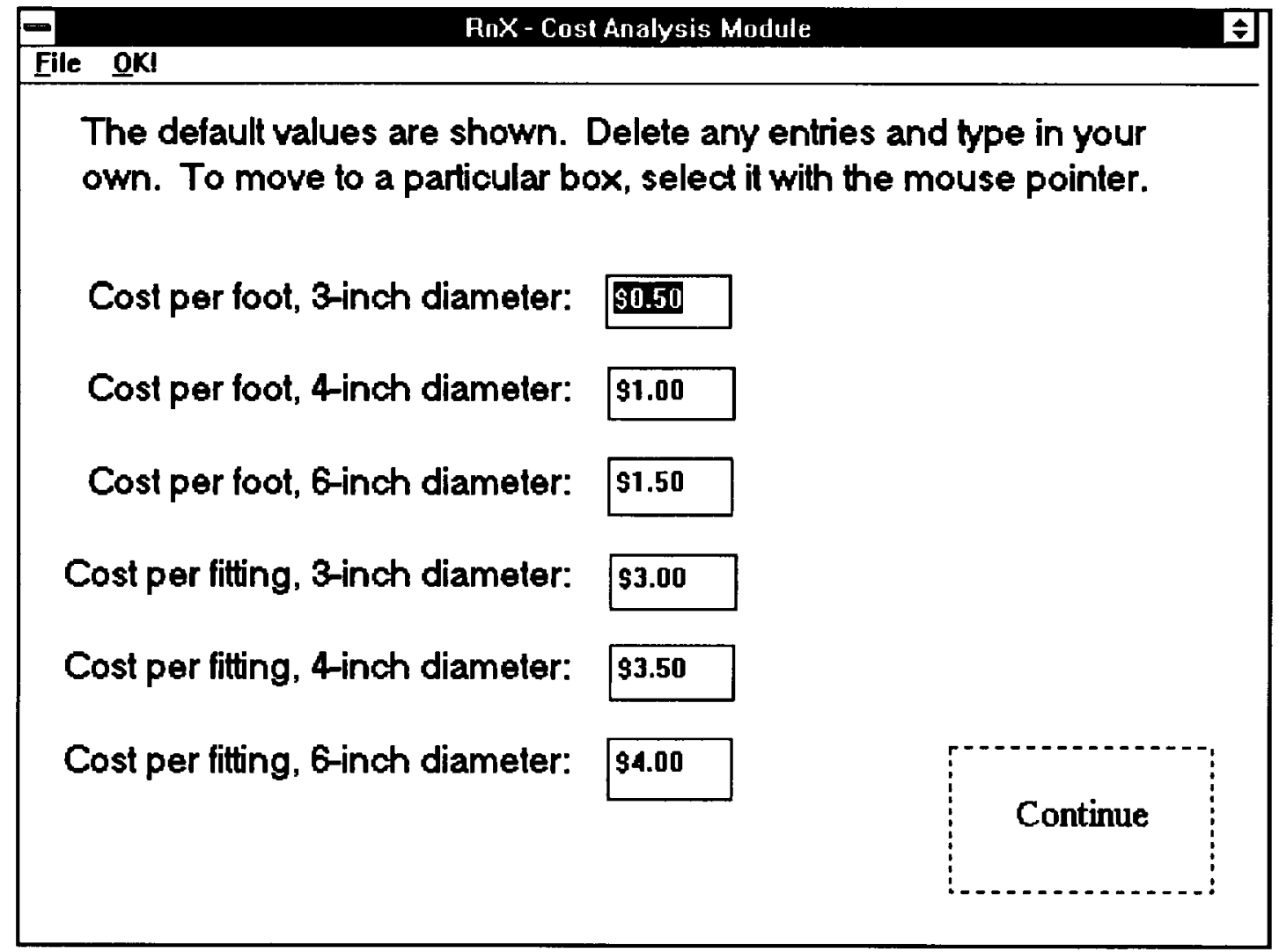

Fiqure 14. Default costs for ducting. 
them. This utility is provided to allow for regional and quality variations in cost. The annual energy cost is based on the fan power consumption, the cost per kilowatt hour, and the assumption that the fan will operate continuously. The power consumption of the fan is obtained along with the fan purchase cost from the Fan selection module. In addition, the user can change the values for a labor cost and the number of hours worked. All of the default values may be changed by the user (see Figure 15).

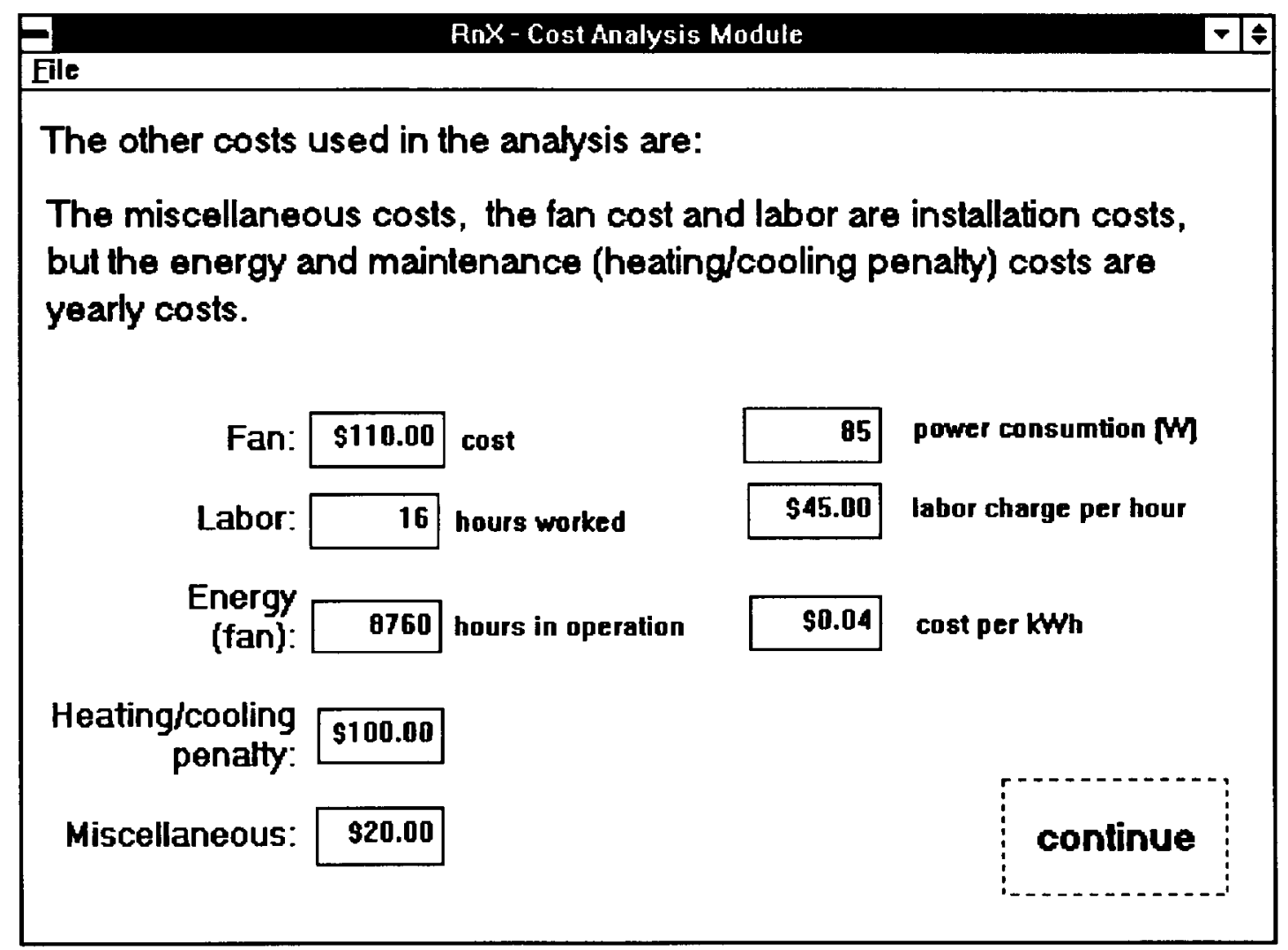

Fiqure 15. Other costs used in estimation.

The cost estimation sums up the material cost for the 
ducting and the fan along with the labor cost and presents it as the installation cost. The energy cost is included in an annual maintenance cost, which includes the heating/cooling penalty due to the year-round operation of the fan, and the cost of miscellaneous items such as sealants, manometers, and alarms.

\section{FAN TUTORIAL}

A fan tutorial module is also available to assist users who are unfamiliar with the format of the regular fan selection module. This module does not depend upon output from a previously executed module; it is completely independent. The tutorial module is in essence a hypertext application, with a rule base derived from the regular fan selection module. However, it is limited in that it can only analyze systems with up to two branches. It does contain more in terms of graphics, the user interface, and on-line explanation referring to diagnostic measurements, the types of fittings that may be used, and the type of ducting configuration. In order to obtain a more detailed explanation about something, a hyperregion labeled "click here for more info" can be activated to access definitions or illustrations. An example screen from the Fan Tutorial module is shown in Figure 16. The fan tutorial module illustrates the potential for a knowledge-based system as a training tool. 
Eile

You have indicated that there are two suction points.

Now the system will require information about the ducting configuration (length, diameter, fittings, etc.).

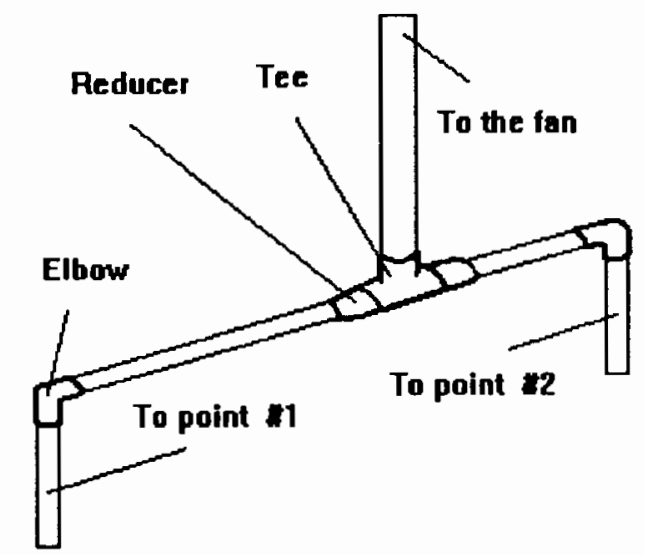

This shows a configuration with two suction points. The diameter of the ducting may vary. The branches that come from the suction points may have different diameters, and the branch that carries their combined flow must have a larger diameter.

Click here to continue

Figure 16. Information presented in tutorial. 
SUMMARY

\section{BENEFITS TO RADON MITIGATORS}

Radon mitigators will benefit from the advisory system in terms of record keeping, observance of established EPA protocol, consistent and accurate fan selection, and cost estimation.

The advisory system writes the house investigation information to a text file. This text file may be recorded on a computer diskette and stored for future reference. The mitigator may wish to retain both the actual house investigation forms and their corresponding text files as a type of back-up system for their business records. Furthermore, the advisory system has the potential to be interfaced with a database. This would facilitate data management and would make it possible for mitigators as well as health and regulatory agencies to keep a database of cases for a particular region. The information contained in such a database may be of use to planners and researchers involved in radon related studies.

The advisory system provides a form of quality assurance for mitigators just entering the field. The advisory system in its final form will have incorporated the suggestions of field experts, and will reflect their 
collective opinion. Therefore, the novice user will have the assurance of the expert opinion, and the client will be reassured that the recommended mitigation method complies with established protocol and professional practice. The Fan Selection module alleviates the amount of computation that must be performed during the design of a ducting system. Since the module also informs the user of potential noise problems, it encourages fine-tuning of the ducting system before the actual installation. This may help to manage the amount of time that is spent on installation, and could possibly eliminate the need for follow-up corrections to ducting systems. Another benefit of the module is that it provides consistent results for similar cases and will prevent possible oversizing of the fan.

The cost Estimation module is an efficient way for the mitigator to provide a quick estimate to the client. It will also assure the client that the quoted price is justified. The module is flexible enough to include unforeseen costs involved in the installation and maintenance of a mitigation system. When used in conjunction with the Fan Selection module, the cost estimations for several different designs may be presented to the client. This would also be a benefit to the client who is deciding between several possible ducting configurations. 
The advisory system can conceivably enhance the professional image of radon mitigation contractors and help to build public trust. It may be compared to the well-known energy audits performed at residences.

\section{FUTURE POTENTIAL}

Aside from assisting established professional mitigators, the advisory system illustrates the potential for a knowledge-based system to serve as an interactive training tool for novice mitigators. A modified version of the software can be developed with the eventual goal of training novice mitigators. A successful training tool should have an uncomplicated and highly visual method of communicating the knowledge to the novice, and a graphical user interface such as the one employed in the advisory system is a fitting representation of this technique. An additional consideration is that the effectiveness of a computerized training tool is reasonably dependent upon the availability of the computers needed to run the software. In small businesses, PC systems have emerged as somewhat more prevalent than their counterparts, and current trends in the PC market are evidence that advanced technology is becoming increasingly available at a lower cost. In addition to analyzing single family residences, the present system could be expanded to incorporate other building types such as schools and commercial offices. 


\section{PROJECTED WORK}

The feasibility of applying expert systems methodology to the problem of indoor radon mitigation has been illustrated by this work. The prototype RnX hybrid advisory system addresses various facets of the radon mitigation problem, from the selection of a mitigation method to the determination of necessary building materials and cost estimation. Efficient modification and the implementation of experts' opinions is facilitated by the modular structure. To date, the system has received favorable reviews after demonstrations at several conferences. Future work remaining on the project includes the addition of recommendations submitted by radon mitigation experts who have been contracted to review the software. 


\section{REFERENCES}

1. Barnes, A. James. "A Nonregulatory Challenge." EPA Journal, volume 12, number 6, August 1986.

2. Sandman, Peter M., Weinstein, Neil D., Klotz, M.L. "Public Response to the Risk from Geological Radon." Journal of Communication, volume 37, Summer 1987.

3. Osborne, Michael C. Radon-Resistant Residential New Construction. U.S. Environmental Protection Agency, EPA-600/8-88-087, Research Triangle Park, NC, July 1988.

4. Duval, Joseph S., otton, James K., Jones, William J. "U.S. Geological Survey, Estimation of Radon Potential in the Pacific Northwest Using Geological Data." Bonneville Power Administration, Contract No. DE-AI79-87BP36431, June 1989.

5. U.S. Environmental Protection Agency. "A Citizen's Guide to Radon." OPA-86-004, Washington DC, August 1986.

6. Residential Radon Contamination And Indoor Air Quality Research Needs. Hearing before the subcommittee on Natural Resources, Agriculture Research And Environment of the Committee on science and Technology, U.S. House of Representatives, 99th Congress, 2nd session, No. 159, September 17, 1986.

7. Mosley, R.B., and Henschel, D.B. Application of Radon Reduction Methods. EPA/625/5-88/024, Air and Energy Engineering Research Laboratory, Research Triangle Park, NC, August 1988.

8. Brennan, T., and Galbraith, S. Practical Radon Control for Homes. Cutter Information Corp., Arlington, MA, 1988 . 
9. Fisk, W.J., et al. "Monitoring and Modeling of Radon Entry into Basements: A Status Report For The Small structures Project." Indoor Environment Program, Applied Science Division and Earth Sciences Division, Lawrence Berkeley Laboratory, LBL-27692, September 1989.

10. Henschel, D.B. Radon Reduction Techniques for Detached Houses, Technical Guidance (Second Edition) . U.S. EPA, EPA/625/5-87/019, Research Triangle Park, NC, January 1988 .

11. U.S. Environmental Protection Agency. "Radon Reduction in New Construction, An Interim Guide." OPA-87009 , August, 1987.

12. U.S. Environmental Protection Agency. "Radon Reduction Methods, A Homeowner's Guide." (Third edition), RD-681, Washington, DC, July 1989.

13. Hitchcock, R.J. "Knowledge-Based System Design Guide Tools." To be included in ASHRAE Transactions, $\mathrm{V}$. 97 Pt. 2, 1991.

14. Mayer, R., Degelman, L.O., Su, C.J., Keen, A., Griffith, P., Huang, J., Brown, D., Kim, Y.S. "A Knowledge-Aided Design System For Energy-Efficient Buildings." To be included in ASHRAE Transactions, V.97, Pt. 2, 1991.

15. Mosley, R.B. Personal communication, 1987.

16. Brambley, M.R., Hanlon, R.L., Parker, G.B. "Expert Systems: A New Approach To Radon Mitigation Training And Quality Assurance." Proceedings, Indoor Air Conference, Toronto, Canada, August 1990, pp 483-487.

17. Brennan, T., and Gillette, L.M. "Interactive House Investigation and Radon Diagnostics Computer Program." Proceedings of the 1990 International Symposium on Radon and Radon Reduction Technology, January 1990.

18. Luger, George F., and stubblefield, William A. Artificial Intelligence and the Design of Expert Systems. The Benjamin/Cummings Publishing Company, Inc., 1989. 
19. Zarefar, H. "An Approach to Mechanical Design Using a Network of Interactive Hybrid Expert Systems." Ph.D. Thesis, University of Texas at Arlington, 1986.

20. LEVEL5 OBJECT User's Guide and Reference Guide. Information Builders, Inc., 1990.

21. U.S. Environmental Protection Agency. Reducing Radon in Structures Manual (Second Edition). U.S. EPA and New York State Energy Office, 1989.

22. Osborne, M.C., Moore, D.G., Southerlan, R.E., Brennan, T., and Pyle, B.E. "Radon Reduction in Crawl Space Houses." Journal of Environmental Engineering, volume 115, number 3, June 1989.

23. Prill, R. Radon Diagnostics and Mitigation Workshop Notes, 1989.

24. ASHRAE Handbook 1981 Fundamentals. American Society of Heating, Refrigerating, And Air-Conditioning Engineers, Inc., 1981. 
APPENDIX A

RNX2 - KNB SELECTED DEMONS AND METHODS 


\section{APPENDIX A}

\section{RNX2.KNB Selected DEMONS and METHODS}

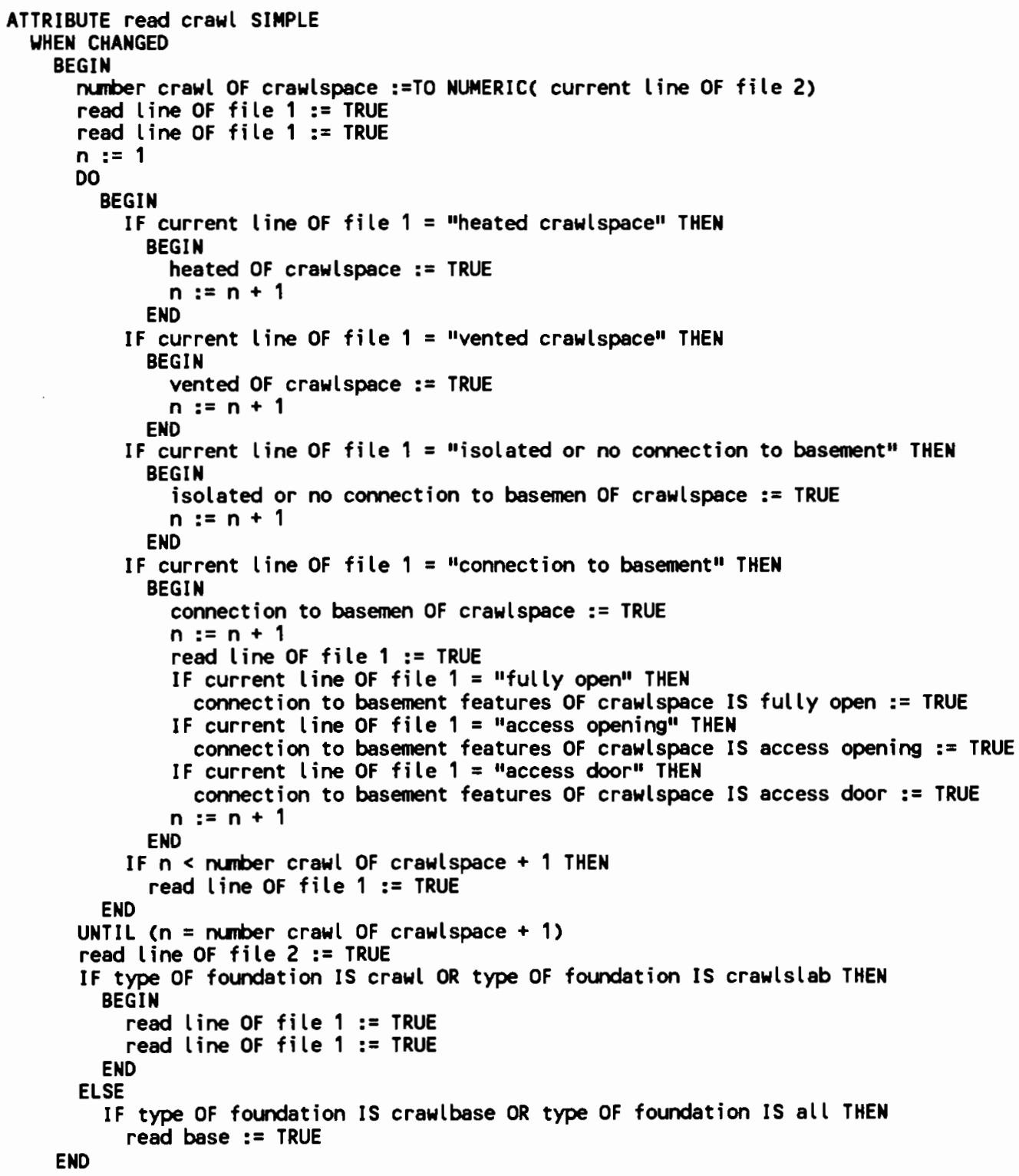




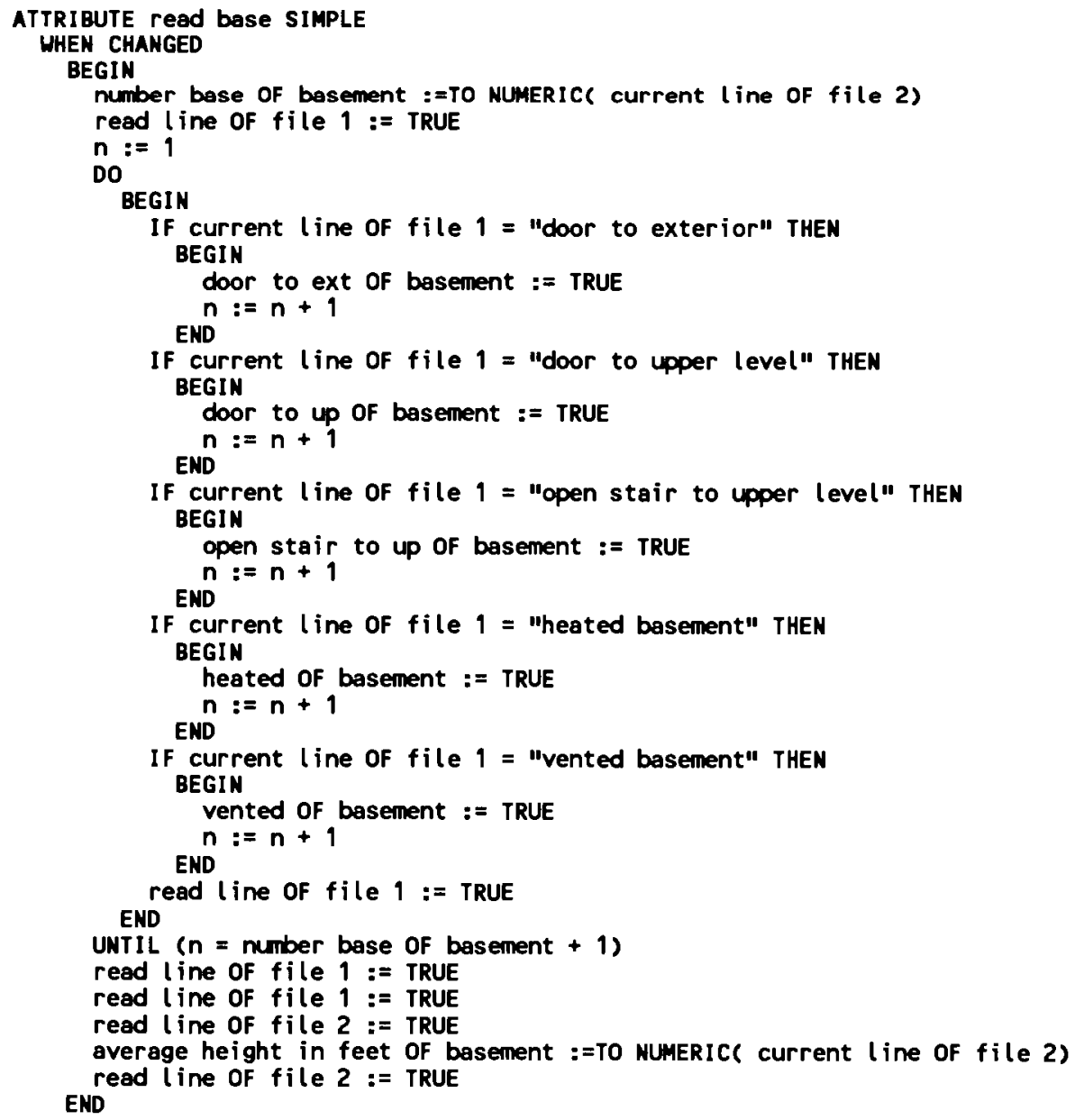

\section{DEMON 41}

If begin processing

AND type OF foundation IS crawl

THEN display attachment Of hyperregion $7:=$ nemmain done 2 display

AND NOT begin processing

AND begin demon processing

\section{DEMON 42}

If begin processing

AND type of foundation Is base

OR type OF foundation IS slab_on_grade

OR type OF foundation IS crawtbase

OR type OF foundation IS craulslab

OR type OF foundation IS baseslab

OR type OF foundation IS all

THEN NOT begin processing

AND begin demon processing

\section{DEMON 1}

If begin demon processing

AND highest result of radon measurements < 4

THEN NOT begin demon processing

AND Not necessary OF mitigation method

AND fill color of textbox $46:=0,255,0$

AND ASK newmain methods display 


\section{DEMON 2}

If begin demon processing

AND highest result OF radon measurements >= 4

THEN NOT begin demon processing

AND get foundation

DEMON 3

IF get foundation

AND type of foundation IS crawl

OR type of foundation IS crawlbase

OR type of foundation IS crawlslab

OR type OF foundation IS all

THEN NOT get foundation

AND start crawl

DEMON 4

IF get foundation

AND type OF foundation Is base

OR type of foundation is bases lab

OR type Of foundation IS slab_on_grade

THEN NOT get foundation

AND start base

DEMON 12

IF start crawl

AND highest result of radon measurements $s=40$

THEN NOT start crawl

AND cont inue 1 crawl

AND ASK newmain crawl 1 display

DEMON 13

If start crawl

AND highest result Of radon measurements $>40$

AND type OF foundation IS crawl

THEN Sub membrane suction of mitigation method

AND write line of file $4:=$ "Sub membrane suction"

AND fill color Of textbox $21:=0,0,255$

AND NOT start crawl

AND done with crawl

DEMON 43

If start crawl

AND highest result of radon measurements $>40$

AND type of foundation Is crawlbase

OR type OF foundation Is crawlslab

OR type OF foundation Is all

THEN Sub membrane suction OF mitigation method

AND fill color of textbox $21:=0,0,255$

AND NOT start crawl

AND done with crawl

DEMON 9

IF permitted to freeze OF crawlspace IS yes

AND cont inue 1 crawl

THEN I solate and ventilate the crawlspace of mitigation method

AND fill color of textbox $22:=0,0,255$

AND NOT cont inve 1 crawl

AND done with crawl

DEMON 10

IF permitted to freeze of crawlspace is no

AND continue 1 crawl

THEN NOT cont inue 1 crawl

AND cont inue 2 crawl

AND ASK newmain crawl 2 display 
DEMON 11

If easy access for barrier of crawlspace is no

AND cont inue 2 crawl

AND vented OF crawl space $=$ FALSE

THEN Crawl space depressurization of mitigation method

AND fill color of textbox $23:=0,0,255$

AND NOT cont inue 2 crawl

AND done with crowl

\section{DEMON 21}

If easy access for barrier Of craulspace is yes

AND cont inue 2 crawl

AND type OF foundation IS crawl

THEN Sub membrane suction of mitigation method

AND write line Of file $4:=$ "Sub membrane suction"

AND fill color of textbox $21:=0,0,255$

AND NOT cont inue 2 crawl

AND done with crawl

\section{DEMON 22}

If easy access for barrier of crawlspace is no

AND cont inue 2 crawl

AND vented OF crawlspace $=$ TRUE

THEN Cranlspace pressurization of mitigation method

AND fill color of textbox $24:=0,0,255$

AND NOT cont inue 2 crawl

AND done with crawl

\section{DEMON 44}

If easy access for barrier of craulspace is yes

AND cont inue 2 crawl

AND type of foundation Is craulbase

OR type OF foundation IS crawlslab

OR type of foundation is all

THEN Sub membrane suction OF mitigation method

AND fill color Of textbox $21:=0,0,255$

AND NOT cont inue 2 crawl

AMD done with crawl

\section{DEMON 7}

If done with crawl

AND type of foundation is crawlslab

THEN NOT done with crawl

AND start base

DEMON 14

If start base

AND $h$ ighest result of radon measurements $>20$

THEN MOT start base

AND cont inue 1 base

DEMON 15

If start base

AND highest result of radon measurements $<=20$

THEN NOT start base

AND continue 2 base

\section{DEMON 17}

If cont inue 1 base

AND NOT interior footer pipe OF drainage

AND NOT exterior footer pipe of drainage

THEN NOT cont inue 1 base

AND continue 3 base 


\section{DEMON 5}

If continue 1 base

AND interior footer pipe of drainage

OR exterior footer pipe of drainage

THEN NOT cont inue 1 base

AND cont inue $3 a$ base

\section{DEMON 26}

If cont inue 2 bese

AND type OF foundation IS slab_on_grade

OR type OF foundation IS crawtslab

THEN NOT cont inue 2 base

AND continue $2 b$ base

\section{DEMON 29}

If continue 2 bese

AND type OF foundation Is base

OR type of foundation is baseslab

THEN NOT cont inue 2 base

AND continue 4a base

\section{DEMON 30}

IF continue 2 base

AND type OF foundation Is crawlbose

$O R$ type OF foundation Is all

THEN NOT cont inue 2 base

AND continue $4 b$ base

\section{DEMON 32}

If cont inue 3 base

AMD degree of sub slab communication Is excellent over entire slab to wall test hole

OR degree OF sub slab communication Is excellent over entire slab

OR degree OF sub slab communication IS fair to good over entire slab

THEN Sub slab suction of mitigation method

AND write line OF file 4 := "Sub slab suction"

AND fill color of textbox $26:=0,0,255$

AND NOT continue 3 base

AND done with base

\section{DEMON 33}

If cont inue 3 base

AND degree OF sub slab cormunication is good at perimeter only

OR degree OF sub slab communication Is marginal

$O R$ degree OF sub slab communication is freon only

OR degree OF sub slab communication IS none observable

AND walls of foundation is block walls

AND NOT open block tops OF potential entry points

THEN Block wall suction OF mitigation method

AND write line of file $4:=$ "Block wall suction"

AND fill color of textbox $27:=0,0,255$

AND NOT continue 3 base

AND done with base

\section{DEMON 34}

If continue 3 base

AND degree Of sub slab communication Is good at perimeter only

OR degree OF sub slab communication is marginal

OR degree of sub slab communication is freon only

OR degree OF sub slab communication Is none observable

AMD walls of foundation is concrete walls

OR walls of foundation is stone walls

OR walls OF foundation is wood walls

THEN Sub slab suction OF mitigation method

AND write line of file 4 : = "Sub slab suction"

AND fill color Of textbox $26:=0,0,255$

AND NOT cont inue 3 base 
AND done with base

\section{DEMON 35}

If cont inue 3 bese

AND degree OF sub slab comminication is good at perimeter only

OR degree OF sub slab commication is marginal

OR degree OF sub slab communication Is freon only

OR degree OF sub slab comminication is none observable

AND walls OF foundation Is block walls

AND open block tops OF potential entry points

THEN Block wall suction after tops are sealed of mitigation method

AND write line of file $4:=$ "Block wall suction"

AND fill color of textbox $44:=0,0,255$

AND NOT continue 3 base

AND done with base

\section{DEMON 25}

If cont inue $3 a$ base

AND sump OF drainage

AND NOT interior pipe OF sump features

AND NOT exterior pipe OF sump features

THEN Drain tile suction of mitigation method

AND fill color of textbox $42:=0,0,255$

AND NOT continue 3 a base

AND done with bese

\section{DEMON 36}

If cont inue $3 a$ bese

AND sump OF drainage

AND interior pipe of sump features

OR exterior pipe of sump features

THEN Drain tile suction on sump OF mitigation method

AND fill color of textbox $43:=0,0,255$

AND NOT continue 3 a base

AND done with base

\section{DEMON 37}

IF cont inue $3 \mathrm{a}$ base

AND NOT sump OF drainage

THEN Drain tile suction of mitigation method

AND fill color of textbox $42:=0,0,255$

AND NOT continue $3 a$ base

AND done with base

\section{DEMON 23}

If continue $2 b$ base

AND present OF airducts = TRUE

THEN NOT cont inve 2 base

AND continue 4 base

\section{DEMON 31}

IF continue $2 b$ base

AND present OF airducts = FALSE

THEN NOT cont inue 2 base

AND cont inue 1 base

\section{DEMON 38}

If continue 4 base

AND climate OF house Is not mild

AND highest OF radon measurements $<10$

THEN Heat recovery ventilation OF mitigation method

AND fill color OF textbox $30:=0,0,255$

AND NOT continue 4 base

AND done with base 
DEMON 39

If continue 4 base

ANO climate OF house is mild

THEN Natural or fan assisted ventilation of house OF mitigation method

AND fill color of textbox $31:=0,0,255$

AND NOT continue 4 base

AND done with base

DEMON 40

If cont inue 4 base

AND climate of house IS not mild

AND highest Of radon measurements $>=10$

THEN NOT cont inue 4 base

AND continue 1 base

DEMON 19

If cont inue $4 \mathrm{a}$ base

AND present OF airducts = FALSE

AND door to up OF basement = FALSE

AND open stair to up OF basement = FALSE

THEN Basement pressurization of mitigation method

AND fill color of textbox $28:=0,0,255$

AND NOT continue $4 a$ base

AND done with base

DEMON 27

If cont inue $4 a$ base

AND present OF airducts = TRUE

OR door to up OF basement = TRUE

OR open stair to up of basement = TRUE

THEN NOT continue $4 \mathrm{a}$ base

AND continue 1 base

DEMON 16

IF continue $4 \mathrm{~b}$ base

AND present OF airducts = TRUE

OR door to up OF basement = TRUE

OR open stair to up OF basement = TRUE

THEN NOT cont inue $4 \mathrm{~b}$ base

AND continue 1 base

DEMON 28

If cont inue $4 \mathrm{~b}$ base

AND present OF airducts = FALSE

AND door to up OF basement = FALSE

AND open stair to up OF basement = FALSE

THEN NOT cont inue $4 \mathrm{~b}$ base

AND cont inue $4 \mathrm{c}$ base

DEMON 20

IF continue $4 \mathrm{c}$ base

AND isolated or no connection to besemen OF crawlspace = FALSE

THEN Not decided OF mitigation method

AND fill color of textbox $47:=255,0,0$

AND NOT continue $4 \mathrm{C}$ base

AND done with base

DEMON 24

If continue $4 \mathrm{c}$ base

AND isolated or no connection to basemen OF crawlspace = TRUE

THEN Basement pressurization of mitigation method

AND fill color of textbox $28:=0,0,255$

AND NOT cont inue $4 \mathrm{c}$ base

AMD done with base 
APPENDIX B

FAN. KNB SELECTED DEMONS 


\section{APPENDIX B}

\section{FAN. KNB Selected DEMONS}

\section{DEMON 1}

If begin of logicals

AND points of numbers $=1$

THEN NOT begin OF logicals

AND ASK fan one1 display

\section{DEMON 2}

If begin of logicals

AND points OF numbers $=2$

THEN NOT begin OF logicals

AND ASK fan twol display

\section{DEMON 3}

If begin of logicals

AND points OF numbers $=3$

THEN NOT begin OF logicals

AND ASK fan three1 display

DEMON 4

If points of numbers $=1$

AND cont inue OF logicals

THEN equivalent length OF onepoint := straight length of onepoint +5 * elbow45 of onepoint +10 * elbows OF onepoint +5 * reducer of onepoint +50 * tee OF onepoint

AND write 4 OF strings

AND find duct loss 1 OF logicals

DEMON 5

If points of numbers $=1$

AND find duct loss 1 of logicals

AND diameter of onepoint $=3$

THEN duct loss OF onepoint $:=\left(10^{\wedge}(1.85 *(\right.$ LOG(flowrate OF onepoint) -1.76$\left.))\right)$ * equivalent length OF onepoint / 100

AND find total $\mathrm{cfm}$ and friction loss OF logicals

\section{DEMON 6}

If points of numbers $=1$

AND find duct loss 1 of logicals

AND dianeter OF onepoint $=4$

THEN duct loss OF onepoint $:=\left(10^{\wedge}(1.85 *(\right.$ LOG(flowrate OF onepoint $\left.\left.)-2.1)\right)\right)$ * equivalent length OF onepoint / 100

AND find total $\mathrm{cfm}$ and friction loss OF logicals

\section{DEMON 7}

If points of numbers $=1$

AND find duct loss 1 of logicals

AND diameter of onepoint $=6$

THEN duct loss OF onepoint $:=(10 \wedge(1.85 *(\operatorname{LOG}($ flowrate OF onepoint $) \cdot 2.76)))$ * equivalent

length OF onepoint / 100

AND find total cfm and friction loss of logicals 
DEMON 8

If points of numbers $=1$

AND find total cfm and friction loss OF logicals

THEN total $c f m$ OF numbers := flowrate OF onepoint

AND NOT find total $\mathrm{cfm}$ and friction loss of logicals

AND total system friction loss OF numbers := duct loss of onepoint + diagnostic friction of onepoint

AND pascals OF numbers := total system friction loss of numbers * 249

AND velocity[ 1] Of velocities := (flowrate of onepoint * 144) / ((dianeter of onepoint $\wedge 2$ ) *

$3.14159 / 4$ )

AND determine max velocity of logicals

DEMON 9

If points of numbers $=2$

AND cont inue of logicals

THEN equivalent length [ 1] OF twopoints := straight length [ 1] of twopoints + 5 * elbows45[ 1] OF twopoints +10 * elbows [ 1] OF twopoints +5 * reducer [ 1] of twopoints +50 * tee [ 1]

AND equivalent length [ 2] OF twopoints := straight length [ 2] of twopoints + 5 * elbows45[ 2] of twopoints of twopoints $+10 *$ elbows [ 2] Of twopoints $+5 *$ reducer [ 2] of twopoints $+50 *$ tee [ 2] OF twopoints

AND write9 of strings

AND find duct loss 21 of logicals

DEMON 10

If points of numbers $=2$

AND find duct loss 21 of logicals

AND diameter [ 1] OF twopoints $=3$

THEN duct loss [ 1] OF twopoints $:=\left(10^{\wedge}(1.85 *(\right.$ LOG(flowrate[ 1] OF twopoints) - 1.76) $)) *$ equivalent length [ 1] of twopoints / 100

AND NOT find duct loss 21 of logicals

AND find duct loss 22 of logicals

\section{DEMON 11}

If points of numbers $=2$

AND find duct loss 21 of logicals

AND diameter[ 1] Of twopoints $=4$

THEN duct loss [ 1] OF twopoints $:=\left(10^{\wedge}(1.85 *(\right.$ LOG $($ flowrate [ 1] OF twopoints $\left.)-2.1))\right) *$ equivalent length [1] OF twopoints / 100

AND find duct loss 22 of logicals

DEMON 12

IF points of numbers $=2$

AND find duct loss 21 of logicals

AND diameter [ 1] OF twopoints $=6$

THEN duct loss [ 1] OF twopoints : $=(10 \wedge(1.85 *($ LOG(flourate[ 1] OF twopoints $)-2.76))) *$ equivalent length [ 1] of twopoints / 100

AND find duct loss 22 of logicals

DEMON 13

If points of numbers $=2$

AND find duct loss 22 of logicals

AND diameter [ 2] OF twopoints $=3$

THEN duct loss [ 2] OF twopoints $:=(10 \wedge(1.85 *($ LOG(flowrate[ 2] OF twopoints) - 1.76) $))$ * equivalent length [ 2] of twopoints $/ 100$

AND find 2 main of logicals

DEMON 14

If points of numbers $=2$

AND find duct loss 22 of logicals

AND dianeter [ 2] OF twopoints $:=4$

THEN duct loss [ 2] OF twopoints $:=(10 \wedge(1.85 *($ LOG $($ flowrate [ 2] OF twopoints $)-2.1)))$ * equivalent length [ 2] OF twopoints / 100

AND NOT find duct loss 22 of logicals

AND find 2 main of logicals 
DEMON 15

If points OF numbers $=2$

AND find duct loss 22 of logicals

AND diameter [ 2] OF twopoints = 6

THEN duct loss [ 2] OF twopoints $:=\left(10^{\wedge}(1.85 *(\right.$ LOG $($ flowrate [ 2] OF twopoints $\left.)-2.76))\right)$ equivalent length [2] Of twopoints / 100

AND find 2 main Of logicals

DEMON 16

If points of numbers $=2$

AND find 2 main OF logicals

THEN equivalent length OF twopoints main branch := straight length of twopoints main branch + 5 * elbow45 Of twopoints main branch + 10 * elbows OF twopoints main branch +5 * reducer of twopoints main branch +50 * tee OF twopoints main branch

AND find duct loss 2 main of logicals

\section{DEMON 17}

If points of numbers $=2$

AND find duct loss 2 main of logicals

AND diameter of twopoints main branch $=3$

THEN duct loss Of twopoints main branch $:=\left(10^{\wedge}\right.$ (1.85* ( LOG(flowrate[ 1] of twopoints + flowrate [ 2] Of twopoints) - 1.76)) * equivalent length Of twopoints main branch $/ 100$

AND find total cfm and friction loss of logicals

\section{DEMON 18}

If points of numbers $=2$

AND find duct loss 2 main of logicals

AND diameter OF twopoints main branch $=4$

THEN duct loss OF twopoints main branch $:=(10 \wedge(1.85 *$ ( LOG(flowrate [ 1] OF twopoints + f(owrate [ 2] Of twopoints) - 2.1)) * equivalent length of twopoints main branch $/ 100$

AND find total $\mathrm{cfm}$ and friction loss of logicals

\section{DEMON 19}

If points of numbers $=2$

AND find duct loss 2 main of logicals

AND diameter of twopoints main branch $=6$

THEN duct loss OF twopoints main branch $:=(10 \wedge(1.85 *$ ( LOG(flowrate[ 1] of twopoints + flowrate [ 2] Of twopoints) - 2.76)) * equivalent length of twopoints main branch / 100

AND find total $\mathrm{cfm}$ and friction loss OF logicals

DEMON 20

If points of numbers $=2$

AND find total $\mathrm{cfm}$ and friction loss OF logicals

THEN total cfm of numbers := flowrate[ 1] OF twopoints + flowrate[ 2] of twopoints

AND NOT find total $\mathrm{cfm}$ and friction loss OF logicals

AND total system friction loss of numbers := diagnostic friction[ 1] of twopoints + diagnostic friction [ 2] of twopoints + duct loss [ 1] Of twopoints + duct loss [ 2] OF twopoints + duct loss of twopoints main branch

AND pescals Of numbers := total system friction loss of numbers * 249

AND velocity [ 2] Of velocities := (flowrate[ 1] Of twopoints $\star 144) /$ ((dianeter [ 1] of twopoints $\wedge$ 2) *3.14159/4)

AND velocity[ 3] OF velocities := (flowrate[ 2] OF twopoints * 144)/ (cdiameter [ 2] OF

twopoints $\wedge$ 2) *3.14159/4)

AND velocity[ 4] Of velocities := ((flowrate[ 1] Of twopoints + flowrate[ 2] of twopoints) * 144) / ((diameter of twopoints main branch $\wedge 2$ ) $\star 3.14159 / 4)$

AND determine max velocity Of logicals

\section{DEMON 21}

If points of numbers $=3$

AND cont inue of logicals

THEN equivalent length [ 1] OF threepoints := straight length [ 1] OF threepoints $+5 \star$ elbow45[ 1] Of threepoints +10 * elbows [ 1] Of threepoints +5 * reducer [ 1] OF threepoints +50 * tee [ 1] OF threepoints

AND equivalent length [ 2] OF threepoints := straight length [ 2] OF threepoints +5 * elbow45 [ 2] Of threepoints +10 * elbows [ 2] of threepoints $+50 *$ tee [ 2] Of threepoints $+5 *$ reducer [ 2] of threepoints 
AND equivalent length [ 3] Of threepoints := straight length [ 3] OF threepoints +5 * elbow45 [ 3] OF threepoints +10 * elbows [ 3] OF threepoints $+50 *$ tee [ 3] OF threepoints $+5 *$ reducer [ 3] OF threepoints

AND write21 OF strings

AND find duct loss 31 of logicals

DEMON 22

If points of numbers $=3$

AND find duct loss 31 of logicals

AND diameter [ 1] Of threepoints $=3$

THEN duct loss [ 1] OF threepoints $:=(10 \wedge(1.85 *(\operatorname{LOG}(f$ lowrate [ 1] OF threepoints) - 1.76) $))$ * equivalent length [ 1] Of threepoints / 100

AND find duct loss 32 of logicals

DEMON 23

If points of numbers $=3$

AND find duct loss 31 of logicals

AND diameter [ 1] OF threepoints $=4$

THEN duct loss [ 1] OF threepoints := $(10 \wedge(1.85 *($ LOG(flowrate [ 1] of threepoints) - 2.1) $)$ * equivalent length [ 1] OF threepoints / 100

AND find duct loss 32 of logicals

DEMON 24

If points of numbers $=3$

AND find duct loss 31 OF logicals

AND diameter [ 1] Of threepoints = 6

THEN duct loss [ 1] OF threepoints $:=(10 \wedge(1.85 *($ LOG(flowrate[ 1] OF threepoints) - 2.76) $))$

* equivalent length [ 1] OF threepoints / 100

AND find duct loss 32 OF logicals

DEMON 25

If points of numbers $=3$

AND find duct loss 32 OF logicals

AND diameter [ 2] Of threepoints $=3$

THEN duct loss [ 2] OF threepoints $:=(10 \wedge(1.85 *($ LOG $(f$ lowrate [ 2] OF threepoints) - 1.76) $))$

* equivalent length [ 2] OF threepoints / 100

AND find duct loss 33 of logicals

DEMON 26

If points of numbers $=3$

AND find duct loss 32 of logicals

AND diameter [ 2] Of threepoints $=4$

THEN duct loss [ 2] OF threepoints $:=(10 \wedge(1.85 *($ LOG $(f$ lowrate [ 2] OF threepoints $)-2.1))) *$ equivalent length [ 2] Of threepoints / 100

AND find duct loss 33 of logicals

DEMON 27

If points of numbers $=3$

AND find duct loss 32 of logicals

AND diameter [ 2] OF threepoints $=6$

THEN duct loss [ 2] Of threepoints := $\left(10^{\wedge}(1.85 *(\right.$ LOG(flowrate[ 2] OF threepoints) - 2.76) $))$

* equivalent length [ 2] Of threepoints / 100

AND find duct loss 33 of logicals

DEMON 28

If points of numbers $=3$

AND find duct loss 33 of logicals

AND diameter [3] OF threepoints $=3$

THEN duct loss [ 3] OF threepoints $:=(10 \wedge(1.85 *($ LOG $($ flowrate[ 3] OF threepoints $)-1.76)))$

* equivalent length [ 3] Of threepoints / 100

AND find 3 intermediate of logicals

DEMON 29

If points of numbers $=3$

AMD find duct loss 33 of logicals 
AND diameter [3] OF threepoints $=4$

THEM duct loss [ 3] OF threepoints $:=\left(10^{\wedge}(1.85 *(\right.$ LOG $(f$ lowrate [ 3] OF threepoints $\left.)-2.1))\right)$ * equivalent length [ 3] of threepoints / 100

AND find 3 intermediate of logicals

DEMON 30

If points of numbers $=3$

AND find duct loss 33 of logicals

AND diameter [3] OF threepoints $=6$

THEN duct loss [ 3] Of threepoints $:=\left(10^{\wedge}(1.85 *(\right.$ LOG $($ flowrate $[3]$ Of threepoints $\left.)-2.76))\right)$

* equivalent length [ 3] Of threepoints / 100

AND find 3 intermediate of logicals

DEMON 31

If points of numbers $=3$

AND find 3 intermediate of logicals

THEN equivalent length OF three interm branch := straight length of three interm branch +5 * elbow45 of three interm branch +10 * elbows Of three interm branch +5 * reducer of three interm branch +50 * tee OF three interm branch

AND find duct loss 3 intermediate of logicals

\section{DEMON 32}

If points of numbers $=3$

AND find duct loss 3 intermediate OF logicals

AND diameter of three interm branch $=3$

THEN duct loss OF three interm branch $:=(10 \wedge(1.85 *$ ( LOG(flowrate[ 1] OF threepoints + flowrate [ 2] OF threepoints) - 1.76)) ) * equivalent length OF three interm branch / 100

AND find 3 main OF logicals

\section{DEMON 33}

If points of numbers $=3$

AND find duct loss 3 intermediate of logicals

AND diameter of three interm branch $=4$

THEN duct loss OF three interm branch $:=(10 \wedge(1.86 *$ ( LOG(flowrate $[1]$ of threepoints + flowrate [ 2] of threepoints) - 2.1)) * equivalent (ength of three interm branch / 100

AND find 3 main of logicals

\section{DEMON 34}

If points of numbers $=3$

AND find duct loss 3 intermediate OF logicals

AND diameter of three interm branch $=6$

THEN duct loss OF three interm branch $:=(10 \wedge(1.85 *$ ( LOG(flowrate[ 1$]$ of threepoints + flowrate[ 2] Of threepoints) - 2.76)) ) * equivalent length of three interm branch / 100

AND find 3 main OF logicals

DEMON 35

If points of numbers $=3$

AND find 3 main OF logicals

THEN equivalent length OF threepoint main branch := straight length of threepoint main branch + 5 * elbow45 Of threepoint main branch +10 * elbow Of threepoint main branch +5 * reducer OF threepoint main branch +50 * tee Of threepoint main branch

AND find duct loss 3 main of logicals

DEMON 36

If points of numbers $=3$

AND find duct loss 3 main of logicals

AND diameter Of threepoint main branch $=3$

THEN duct loss OF threepoint main branch $:=\left(10^{\wedge}(1.85 *\right.$ ( LOG(flowrate [ 1] OF threepoints + flowrate [ 2] OF threepoints + f(owrate [ 3] OF threepoints) - 1.76)) ) * equivalent (ength of threepoint main branch / 100

AND find total cfm and friction loss of logicals

DEMON 37

If points of numbers $=3$

AND find duct loss 3 main of logicals 
AND diameter OF threepoint main branch $=4$

THEN duct loss OF threepoint main branch $:=\left(10^{\wedge}(1.85 *\right.$ ( LOG(flowrate[ 1] OF threepoints + flowrate [ 2] of threepoints + f(owrate[ 3] Of threepoints) - 2.1)) ) * equivalent (ength of threepoint main branch / 100

AND find total cfm and friction loss OF logicals

\section{DEMON 38}

If points of numbers $=3$

AND find duct loss 3 main of logicals

AND diameter of threepoint main branch $=6$

THEN duct loss OF threepoint main branch $:=\left(10^{\wedge}(1.85 *\right.$ ( LOGCflowrate[ 1] OF threepoints + flowrate [ 2] OF threepoints + flowrate [ 3] OF threepoints) - 2.76)) ) * equivalent length of threepoint main branch / 100

AND find total $\mathrm{cfm}$ and friction loss of logicals

\section{DEMON 39}

If points of numbers $=3$

AND find total $\mathrm{cfm}$ and friction loss OF logicals

THEN total cfm OF numbers := flowrate [ 1] OF threepoints + flowrate [ 2] OF threepoints + flowrate [ 3] OF threepoints

AND NOT find total $\mathrm{cfm}$ and friction loss OF logicals

AND total system friction loss OF numbers := diagnostic friction [ 1] OF threepoints + diagnostic friction [ 2] Of threepoints + diagnostic friction [3] OF threepoints

AND total system friction loss OF numbers := total system friction loss OF numbers + duct loss [ 1] OF threepoints + duct loss [ 2] OF threepoints + duct loss [ 3] OF threepoints

AND total system friction loss Of numbers := total system friction loss of numbers + duct loss OF three interm branch + duct loss Of threepoint main branch

AND pascals Of numbers : = total system friction loss of numbers * 249

AND velocity[ 5] OF velocities := (flowrate[ 1] OF threepoints * 144) / ((diameter [ 1] OF threepoints $(2) * 3.14159 ; 4$ )

AND velocity[ 6] Of velocities := (flowrate[ 2] OF threepoints * 144) / (cdiameter [ 2] OF threepoints ${ }^{\wedge}$ 2) $* 3.14169 ; 4$ )

AND velocity[ 7] OF velocities := (flowrate[ 3] OF threepoints * 144) / ((diameter [ 3] OF threepoints $\wedge$ 2) * $3.14159,4$ )

AMD velocity [ 8] Of velocities : = ((flowrate [ 1] OF threepoints + flowrate [ 2] Of threepoints) $* 144)$ / ((diameter Of three interm branch $\left.{ }^{\wedge} 2\right) * 3.14159 / 4$ )

AND velocity[ 9] OF velocities := ((flowrate[ 1] OF threepoints + flowrate[ 2] OF threepoints + flowrate [ 3] OF threepoints) * 144) / ((diameter Of threepoint main branch ^ 2) *3.14159/, 4)

AND determine max velocity of logicals

DEMON 40

If determine fan1 of logicals

AND total system friction loss of numbers $s=(-0.9 / 122) *$ total cfm of numbers +0.9

THEN NOT determine fan 1 OF logicals

AND type 1 OF fan IS $K 4$

AND action of file 3 is close

AND determine fan2 of logicals

\section{DEMON 41}

If determine fan1 of logicals

AND total system friction loss Of numbers $s=(-0.92 / 157)$ * total $\mathrm{cfm}$ OF numbers +0.92

AND total system friction loss of numbers $>(-0.9,122)$ * total cfm Of numbers +0.9

THEN NOT determine fan 1 OF logicals

AND typel of $f$ an IS $K 5$

AND action OF file 3 IS close

AND attachment OF valuebox $10:=$ type 1 power [ 2] OF fan costs

AND attachment OF valuebox $11:=$ typel cost [ 2] OF fan costs

AND determine fan2 of logicals

\section{DEMON 42}

If determine fan 1 of logicals

AND total system friction loss OF numbers $s=(-1.7 / 260)$ * total cfm of numbers +1.7

AND total system friction loss of numbers $>(-0.92 / 157)$ * total cfm of numbers +0.92

THEN NOT determine fan 1 OF logicals

AND typel OF fan IS K6

AND action OF file 3 is close

AND attachment OF valuebox $10:=$ type1 power [3] OF fan costs 
AND attechment OF valuebox $11:=$ type1 cost [ 3] OF fan costs AND determine fan2 OF logicals

DEMON 43

If determine fant of logicals

AND total system friction loss of numbers $<=(-2.4 / 510) *$ total cfm of numbers +2.4

AND total system friction loss OF numbers $>(-1.7 / 260)$ * total cfm of numbers +1.7

THEN NOT determine fanl of logicals

AND type1 of fan $15 \mathrm{~KB}$

AND action of file 3 is close

AND attachment OF valuebox $10:=$ type1 power [ 4] of fan costs

AND attachment OF valuebox $11:=$ type1 cost [ 4] OF fan costs

AND determine fane of logicals

DEMON 44

If determine fan1 of logicals

AND total system friction loss OF numbers $<(-2.9 / 630)$ * total cfm of numbers +2.9

AND total system friction loss of numbers $>(-2.4,510)$ * total cfm of numbers +2.4

THEN NOT determine fanl OF logicals

AND type 1 of fan $15 \times 10$

AND action of file 3 is close

AND attachment OF valuebox $10:=$ type1 power [ 5] Of fan costs

AND attachment Of valuebox $11:=$ type 1 cost [ 5] of fan costs

AND determine fanz of logicals

DEMON 45

If determine fan1 of logicals

AND total system friction loss OF numbers $s=(-3.7 / 795)$ * total cfm of numbers +3.7

AND total system friction loss of numbers $>(-2.9 / 630)$ * total cfm of numbers +2.9

THEN NOT determine fan1 OF logicals

AND type 1 of fan IS K12

AND action of file 3 is close

AND attachment of valuebox $10:=$ type1 power [ 6] of fan costs

AND attachment OF valuebox $11:=$ typel cost [ 6] Of fan costs

AND determine fan2 of logicals

DEMON 46

If determine fanl of logicals

AND total system friction loss of numbers $>(-3.7 / 795) *$ total cfm of numbers +3.7

THEN NOT determine fan 1 of logicals

AND type 1 OF fan is Larger than $\mathrm{K} 12$

AND action of file 3 is close

AND attachment OF valuebox $10:=$ type 1 power [ 7$]$ of fan costs

AND attachment of valuebox $11:=$ type 1 cost [ 7] of fan costs

AND determine fanz of logicals 